\title{
A Critical Review on Pulsed Electric Field: A Novel Technology for the Extraction of Phytoconstituents
}

\author{
Muhammad Modassar A. N. Ranjha ${ }^{1}{ }^{(0)}$, Rabia Kanwal ${ }^{1}{ }^{\circledR}$, Bakhtawar Shafique $^{1}$, Rai Naveed Arshad ${ }^{2}$, \\ Shafeeqa Irfan $\left.{ }^{1}{ }^{(}\right)$, Marek Kieliszek ${ }^{3, *} \mathbb{C}^{\mathbb{D}}$, Przemysław Łukasz Kowalczewski ${ }^{4}{ }^{\circ}$, Muhammad Irfan ${ }^{5}$, \\ Muhammad Zubair Khalid ${ }^{6}\left(\mathbb{D}\right.$, Ume Roobab ${ }^{7}\left(\mathbb{D}\right.$ and Rana Muhammad Aadil ${ }^{8, *(1)}$
}

check for updates

Citation: Ranjha, M.M.A.N.; Kanwal, R.; Shafique, B.; Arshad, R.N.; Irfan, S.; Kieliszek, M.; Kowalczewski, P.Ł.; Irfan, M.; Khalid, M.Z.; Roobab, U.; et al. A Critical Review on Pulsed Electric Field: A Novel Technology for the Extraction of

Phytoconstituents. Molecules 2021, 26, 4893. https://doi.org/10.3390/ molecules26164893

Academic Editor: Francisco J. Barba

Received: 6 July 2021

Accepted: 11 August 2021

Published: 12 August 2021

Publisher's Note: MDPI stays neutral with regard to jurisdictional claims in published maps and institutional affiliations.

Copyright: (c) 2021 by the authors Licensee MDPI, Basel, Switzerland. This article is an open access article distributed under the terms and conditions of the Creative Commons Attribution (CC BY) license (https:// creativecommons.org/licenses/by/ $4.0 /)$.
1 Institute of Food Science and Nutrition, University of Sargodha, Sargodha 40100, Pakistan; modassarranjha@gmail.com (M.M.A.N.R.); kanwalrabia043@gmail.com (R.K.); bakhtawarShafique111@gmail.com (B.S.); shafeeqairfan@gmail.com (S.I.)

2 Institute of High Voltage and High Current, Faculty of Engineering, Universiti Teknologi Malaysia, Skudai 81310, Johor, Malaysia; rainaveed77@gmail.com

3 Department of Food Biotechnology and Microbiology, Institute of Food Sciences, Warsaw University of Life Sciences-SGGW, Nowoursynowska 159 C, 02-776 Warsaw, Poland

4 Department of Food Technology of Plant Origin, Poznań University of Life Sciences, 31 Wojska Polskiego St. 60-624 Poznań, Poland; przemyslaw.kowalczewski@up.poznan.pl

5 Department of Food Engineering, University of Agriculture, Faisalabad 38000, Pakistan; ikhan7791@outlook.com

6 Department of Food Science, Government College University, Faisalabad 38000, Pakistan; zubairkhalid730@gmail.com

7 School of Food Science and Engineering, South China University of Technology, Guangzhou 510006, China; mahroba73@gmail.com

8 National Institute of Food Science and Technology, University of Agriculture Faisalabad, Faisalabad 38000, Pakistan

* Correspondence: marek_kieliszek@sggw.edu.pl (M.K.); dilrana89@gmail.com (R.M.A.)

\begin{abstract}
Different parts of a plant (seeds, fruits, flower, leaves, stem, and roots) contain numerous biologically active compounds called "phytoconstituents" that consist of phenolics, minerals, amino acids, and vitamins. The conventional techniques applied to extract these phytoconstituents have several drawbacks including poor performance, low yields, more solvent use, long processing time, and thermally degrading by-products. In contrast, modern and advanced extraction nonthermal technologies such as pulsed electric field (PEF) assist in easier and efficient identification, characterization, and analysis of bioactive ingredients. Other advantages of PEF include cost-efficacy, less time, and solvent consumption with improved yields. This review covers the applications of PEF to obtain bioactive components, essential oils, proteins, pectin, and other important materials from various parts of the plant. Numerous studies compiled in the current evaluation concluded PEF as the best solution to extract phytoconstituents used in the food and pharmaceutical industries. PEF-assisted extraction leads to a higher yield, utilizes less solvents and energy, and it saves a lot of time compared to traditional extraction methods. PEF extraction design should be safe and efficient enough to prevent the degradation of phytoconstituents and oils.
\end{abstract}

Keywords: PEF; green extraction techniques; phytochemical extraction; oil extraction; food waste

\section{Introduction}

Plants with complex structures contain phenolic compounds in cell vacuoles and a lipoprotein bilayer. However, the membrane envelope controls intracellular movement within the intact cells [1-6]. From medieval cultures, natural products and food have been extracted as an essential source of spiritual, cosmetic, and nutritious ingredients. The Pharaonic civilization (more than 4000 years ago) was the first to use solid-liquid extraction to separate aromas, colors, and other materials from plants [7]. Soxhlet, heat reflux, soaking, water percolation, maceration, magnetic stirrer, boiling, and grinding 
are the traditional practices used to obtain phytoconstituents but involve high solvent consumption, a long processing time, poor or low extraction yields, and few have thermal degradation risks. To overcome the limitations of these traditional technologies, numerous innovative non-thermal extraction techniques have been evaluated [8,9].

Pulsed electric field (PEF) has gained more attention in the past few years to extract beneficial materials from food waste/by-products through diffusion, osmosis, pressing, and drying [10]. It minimizes the deleterious effects of conventional heating processes [11-13]. PEF technology, as a promising alternative to other methods (boiling, microwave- and ultrasound-assisted extractions, etc.), has been used for the successful separation, intensification, stabilization, and dehydration of important compounds without affecting the nutritive properties [14-17]. Apart from improving the extraction, PEF has been recently proposed as a tool to induce stress in plant cells, thus stimulating the biosynthesis of active components [18].

Due to its ability to electroporate cell membrane, PEF is used as a pre-treatment to facilitate the recoveries of bioactive material followed by a subsequent traditional or novel extraction step [19]. The PEF method, when applied on water, showed a reduced temperature while consuming less solvent, and enhancing the rate at which the constituents were extracted [20]. PEF has also been used for recycling food waste and by-products through extracting valuable ingredients [21]. It decreased energy costs, improved the extraction yield, lessened the degradation of heat-sensitive substances, and purified extraction with no environmental impact [15]. The current review confines the latest research on PEF assisted extraction focusing on its types, mechanism, and applications in the food industry.

\section{Working Principle of PEF-Assisted Extraction}

The PEF technique uses moderate to high electric field strength (EFS) ranging from $100-300 \mathrm{~V} / \mathrm{cm}$ in batch mode and $20-80 \mathrm{kV} / \mathrm{cm}$ in continuous mode extraction. Two views are common among various hypotheses concerning the potential PEF mechanism. In the biological cell membrane, one is the speeding of chemical-based reactions from various compounds to enhance the solubility of solvent [22] and the other is the electroporation process. Electro-permeabilization or electroporation involves an external electrical force, which enhances the permeability of cell membranes [23]. Food or any other targeted materials are placed between the electrodes and a high-voltage electric field. The cell membrane is punctured by creating hydrophilic pores, which opened protein channels. The sample experiences a force per unit charge called the electric field when high-voltage electrical pulses are applied through the electrodes. The membrane loses its structural functionality, and the plant material is extracted [24]. This mechanism has been shown in Figure 1.

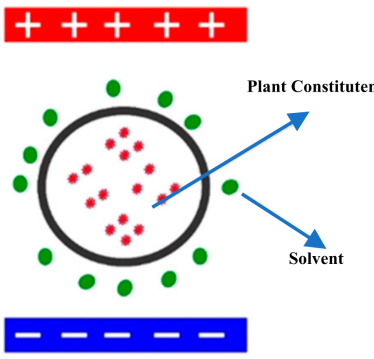

Cell
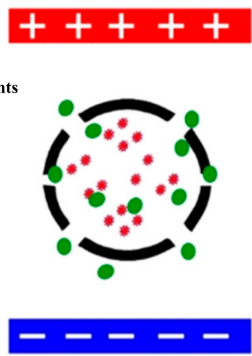

Cell Raptured

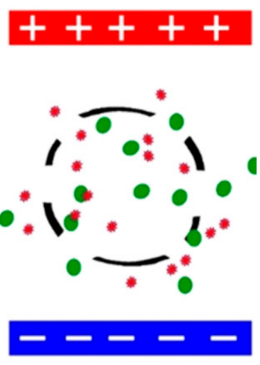

Plant Constitutents

Figure 1. Electroporation mechanism for extraction.

An electric field can be applied either in exponentially decaying, oscillatory square waves, unipolar triangular, or bipolar pulses. The electroporation that occurs is either reversible or irreversible, but depending on the application, this effect can be controlled [25]. Generally, low specific energy (1-10 kJ/kg) and the time (nanoseconds to milliseconds) of the pulses cycle are efficient during the extraction process [26]. The electroporation 
of eukaryotic and prokaryotic cells and the formation of irreversible (permanent) and reversible (temporary) pores in their cell membranes were observed during the extraction process [27]. Irreversible electroporation increases the extraction process. However, EFS increases the cell membrane permeability, which depends on the size of the cell and cell geometry [28]. The strength of an electric field ranging from 0.1 to $10 \mathrm{kV} / \mathrm{cm}$ is enough for delicate plant tissues (e.g., pericarp or mesocarp of few fruits); however, tough materials such as seeds need high intensities (i.e., 10 to $20 \mathrm{kV} / \mathrm{cm}$ ) for efficient extraction [29]. This also provides some additional benefits such as preserving the nutritional, and sensory characteristics of liquid foods [30].

The cells subjected to an external electric field exhibited a high transmembrane potential due to the accumulation of charge on the surface of the membrane. To protect the membrane, a cell has an electrical endurance limit, i.e., cell membranes can endure a specific electrical field strength without considerable damage. The critical electric field is the smallest threshold value for the same cell type. This will facilitate the development of pores in vulnerable areas of the membrane that can be reversible or permanent, depending on the strength of the electric field. The EFS is directly proportional to the magnitude of the damage. Damage is reversible (temporary) when the intensity is low to moderate (not much greater than the critical value). An electric field of high intensity caused irreversible (permanent) damage to the cell membrane [31]. Accordingly, PEF enhances the mass transfer by demolishing the structure of the cell membrane [22]. The increased membrane permeability led to cell breakdown, while the increased rate of mass transfer assisted the discharge of intracellular substances [32]. The degree of electroporation depends on the EFS, type and number of pulses waveform, treatment time, and targeted plant material [33]. However, the specific EFS depends on the geometry and distance of the electrodes.

\section{PEF-Assisted Extraction Equipment}

A PEF unit consists of a high-voltage pulse generator, a treatment chamber with a fluid managing assembly and a monitoring and control system [34]. Additionally, PEF equipment has a charger (to convert $\mathrm{AC}$ in $\mathrm{DC}$ ) and a device that stores energy in the generator. A high-voltage circuit is switched on and off to generate electric pulses. During the discharge of high electric energy, the high-voltage value and short time pulses make this process more complicated, while the capacitor is continuously monitored and stepped up if the voltage is interrupted [35]. The treatment chamber consists of two separate electrodes (one electrode attached to the high-voltage generator and the other to the ground) and a gap; filled with the targeted food. The different electric potential on either side of the membrane generates an electric field, which depends on the electrodes type and the distance among them and with the sample. Other factors include the electric pulses nature, the configuration of the treatment chamber, and the product's conductivity [28]. The PEF treatment parameters required to enhance polyphenols extraction, based on empirical experience, are categorized as high $\left(\mathrm{E}>1 \mathrm{kV} \mathrm{cm}^{-1}\right)$, medium $\left(\mathrm{E} \approx 0.1-1 \mathrm{kV} \mathrm{cm}^{-1}\right)$, and low $\left(\mathrm{E}<0.1 \mathrm{kV} \mathrm{cm}^{-1}\right)$ electric fields [35]. Generally, the PEF extraction process can be grouped into the following two broad categories: one is batch, and the other is a continuous system of extraction concerning the way of operation [22].

\subsection{PEF Batch Extraction}

A standard batch extraction unit comprises a pretreatment chamber for PEF and essential equipment for solid-liquid extraction. The pretreatment unit of PEF contains a cylindrical vessel of polypropylene ( $30 \mathrm{~mm}$ diameter on the inner side), having two stainless steel electrodes arranged parallel to each other at a 10-millimeter distance. The processing parameters include electrical field strength, pulses width, shape, number, and frequency. Inside the cylindrical unit, the sample with a little solvent was first treated between two electrodes and connected to a PEF generator. The treated sample was removed from the cylindrical unit and stirred at different velocities using a magnetic agitator to prevent solution evaporation [22]. 
Using a PEF batch extraction method, various intracellular compounds such as lipid [36], red beetroot pigment [37], anthocyanin [35], betanine [22], oil [38], polyphenols [31], and cellulose [39] have been extracted. Figure 2 shows the PEF batch extraction system.

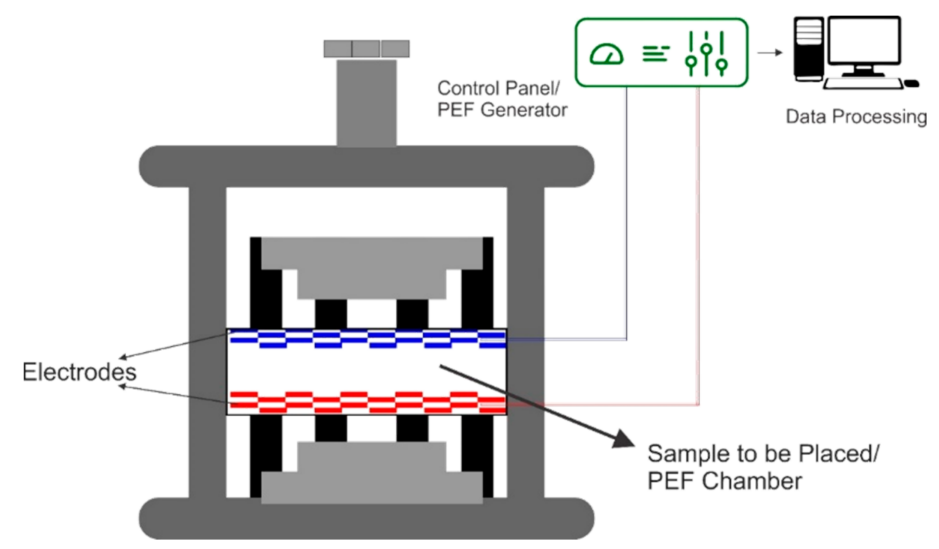

Figure 2. PEF (pulsed electric field) batch extraction system.

\subsection{PEF Continuous Extraction}

The batch extraction process of PEF gave promising results in terms of extraction; however, a significant increase in the operating time has been observed, which is due to the low capacity of batch mode systems. Therefore, it is crucial to devise a PEF extraction process to perform it in regular conditions at the industrial level. Since then, the PEF continuous extraction method has been in great focus. The continuous-flow treatment chamber was first successfully used by Yongguang et al. [35] for the extraction of polysaccharides from Rana temporaria chensinensis David in 2006 [40]. Their results depicted that the extraction yield was $55.59 \%$ with PEF $(20 \mathrm{kV} / \mathrm{cm}, 0.5 \%$ of $\mathrm{KOH})$ as compared to the traditional extraction technique. In the past few years, the continuous extraction PEF applied successfully in laboratories to obtain fishbone, broccoli juice, eggshell, tomato juice, etc. This technology has been applied in industries thus far.

A typical PEF continuous extraction system consists of a high-voltage pulse generator, a treatment chamber, a suitable product handling system, and a range of monitoring and controlling equipment. The oscilloscope can read the output voltage (up to $40 \mathrm{kV}$ pulse voltage) directly, and the frequency is adjustable $(40-3000 \mathrm{~Hz})$. During continuous extraction PEF, the solvent mixture is pumped into the treatment chamber by a peristaltic pump at a constant fluid velocity. The cooling coil has a temperature of $25{ }^{\circ} \mathrm{C}$ in a water bath controlled by a thermostat during the extraction procedure. Coaxial and the cofield continuous PEF treatment chambers are currently widely used due to their simple configurations [22]. Figure 3 shows the PEF continuous extraction system.

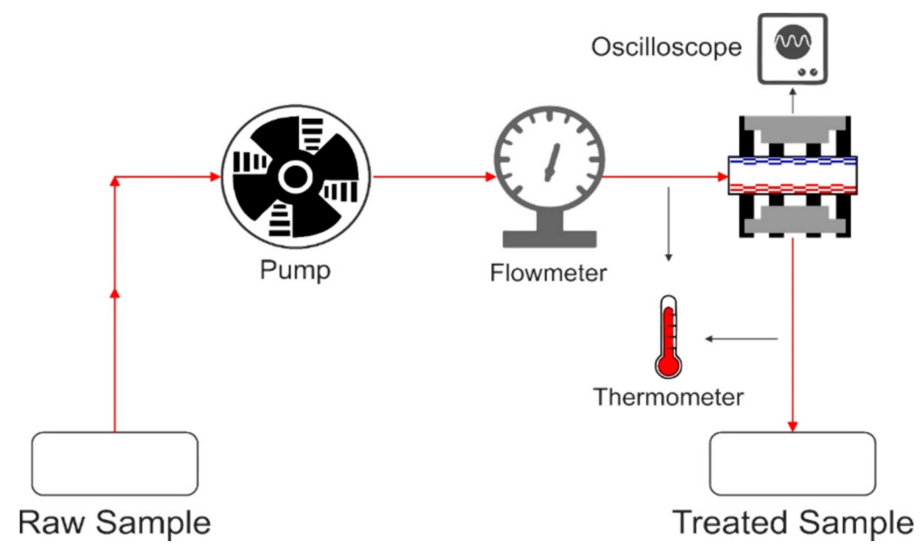

Figure 3. PEF (pulsed electric field) continuous extraction system. 


\section{Factors Influencing the PEF Extraction}

The efficacy of the PEF extracting system depends not only on the processing parameters, but also on the solvent nature, sample composition (size, shape, $\mathrm{pH}$, conductivity, etc.), and the extracted component's size and position in the cell cytoplasm or vacuoles. Moreover, the characteristics of the tissues and cells have a powerful impact on the efficiency of PEF-assisted extraction [28,41]. The extraction process improved in the low ionic strength. The ionic strength affects the cytoplasmic system in terms of the cell's compression and electroporation. However, the conductivity of the matrix affects the behavior of the electric field that passes through that matrix significantly [42].

EFS is a significant parameter in determining the degree of extraction as it affects the physical properties of the targeted compound such as diffusivity, surface tension, viscosity, and solubility [14]. The critical point is to assure the uniform distribution of electric fields across the treatment chamber. Electric field energy delivers through bipolar, exponential decaying, oscillatory, and square pulses. Among them, exponential square wave pulses are widely used in the PEF extraction process due to their high energy and lethal performance. Moreover, square waves are the most prevalent waveform used for the extraction process.

Generally, 12 to $45 \mathrm{kV} / \mathrm{cm}$ is enough to extract the valuable components from food; however, the PEF intensity depends on the food characteristics. Similarly, the extraction of target compounds is improved with an increase in the EFS due to high energy transfer in the food sample. Moreover, the PEF intensity $(5-10 \mathrm{kV} / \mathrm{cm})$ increased the hydrolysis rate and the total amino nitrogen content of abalone viscera protein. The results showed that the mild PEF intensity caused the cell membrane to undergo reversible electroporation. Hence, mild PEF intensity strength reclosed the cell electric hole. However, a PEF intensity greater than $10 \mathrm{kV} / \mathrm{cm}$ exudated the intracellular material due to an irreversible breakdown of the cell membrane. According to the authors, $20 \mathrm{kV} / \mathrm{cm}$ was the optimal EFS for a better extraction of bioactive compounds [43]. Lin et al. [44] found a significant increase $(5.042 \pm 0.04$ to $6.996 \pm 0.03 \mathrm{mg} / \mathrm{mL})$ in the calcium malate extraction from an eggshell using PEF at 0 to $10 \mathrm{kV} / \mathrm{cm}$ EFS. According to the authors, the PEF treatments accelerated the movements between ionic groups and electrons having more kinetic energy, which increased the extraction rate of calcium malate and malic acid. However, the higher EFS facilitated the dissolution of calcium ions. Alternatively, when the pulse time raised from 16 to $20 \mathrm{~s}$, the content of dissoluble calcium malate was significantly reduced. They further explained that the narrow pulses excited the material to resonance vibration. It was also established that, the extreme high EFS, i.e., $35 \mathrm{kV} / \mathrm{cm}$, caused the vitamin $\mathrm{C}$ degradation of broccoli juice [14].

Treatment temperature is another important factor affecting the PEF extraction process [45]. PEF extraction technology is a nonthermal process, thus it operates in room or near room temperature. Higher temperatures usually decrease the viscosity of liquid solvents, which is destructive for the extraction process. Treatment time (pulse numbers and width) is another parameter to measure PEF efficiency [42]. However, an increase in treatment time could raise the temperature of the product. According to the results, longer pulses maximized the extraction rate of polyphenols from fresh tea leaves at $0.9 \mathrm{kV} / \mathrm{cm}$ for $0.5 \mathrm{~s}$ and $1.1 \mathrm{kV} / \mathrm{cm}$ for $3 \mathrm{~s} \mathrm{[46].} \mathrm{Other} \mathrm{than} \mathrm{that,} \mathrm{the} \mathrm{selection} \mathrm{of} \mathrm{solvent} \mathrm{is} \mathrm{also} \mathrm{crucial} \mathrm{for}$ better PEF extraction. It involves numerous factors such as the solubility, conductivity, and polarity of the solvent. The increased solvent conductivity enhanced the cell membrane electroporation that ultimately improved the extraction rate. Similarly, the high solubility of the extract in the solvent and the strong polarity of the solvent increased the mass transfer rate and extraction rate [47-49].

\section{Applications}

\subsection{Fruits and Vegetables}

Due to their nutritional value worldwide and the fact that they are rich sources of beneficial antioxidants, minerals, vitamins and fibers [50], fruits, and vegetables are the most common source of nutrition. A variety of antioxidant compounds can be present in 
fruits and vegetables, including phenolics, carotenoids, anthocyanins, and tocopherols [51]. PEF is a promising method to extract bioactive compounds (anthocyanins, betanines, carotenoids, etc.) from fruit and vegetables, as shown in Table 1. Several factors depend on the extraction of bioactive compounds, such as the process of extraction, the solvent used for extraction and raw materials [52]. Traditional extraction techniques such as Hydro distillation, maceration, and Soxhlet require agitation, high temperatures, and chemical or organic solvents [53]. Nevertheless, the Soxhlet method needs considerable time for extraction and significant quantities of the solvent [54].

Leong et al. [55] evaluated the extraction of anthocyanins from grape juices using PEF technology with $20 \mu \mathrm{S}$ pulse, $50 \mathrm{~Hz}$ frequency and $1.5 \mathrm{kV} / \mathrm{cm}$ electric field power. PEFassisted extraction improved the release of vitamin $C$ and anthocyanins as well as enhanced the antioxidant activity of grape juice as compared to untreated samples. The resultant extract had good phytochemical composition and protected the cells from oxidation [55]. Similarly, the PEF treatment enhanced the antioxidant activity (1.03 times) and extraction of polyphenols (1.44 times) of green grape juice [56]. Moreover, the PEF pretreatment enhanced the recovery of red raspberry (Rubus idaeus L.) juice by $9-25 \%$ without affecting the total phenolic and anthocyanin content. Furthermore, it enhanced the red raspberry press-cake extract that involved a $20 \%$ increase in phenolic content and a $26 \%$ increase in anthocyanin content [57]. In another study, PEF $(13.3 \mathrm{kV} / \mathrm{cm}, 0-564 \mathrm{~kJ} / \mathrm{kg})$ enhanced the extraction yields of fermented grape (Dunkelfelder) pomace up to 22 and $55 \%$. The results showed more recovery of anthocyanins as compared to traditional grinding methods, ultrasound-assisted extraction, and high-voltage electrical discharge [58].

PEF treatment is the most suitable nonthermal technique to extract phenols and flavonoids from onions without significant quality losses. Plant tissues contain a cytomembrane, which affects the movement of intracellular substances between cells. PEF treatments altered the functionality (permeability) of cytomembrane by disintegration and improved the movement of mass through cells, providing greater yields. Compared to the control samples, the PEF treatment significantly enhanced the phenolic compounds $(102.86 \mathrm{mg}$ GAE/100 g) by 2.2 times and the flavonoid compounds ( $37.58 \mathrm{mg} Q E / 100 \mathrm{~g}$ ) by 2.7 times in onions [59]. According to Fincan [60], the disintegration index was $0.86 \pm 0.02$ at 99 pulses of $3 \mathrm{kV} / \mathrm{cm}(4102 \pm 239 \mathrm{~J} / \mathrm{kg})$ in the PEF-treated sesame seed cake. The extraction yields (total phenolic, antioxidant power, and antioxidant activity) produced using microwave and heat technology was comparable with PEF-assisted extraction [60]. Further, Sarkis et al. [61] stated that the disintegration index and the proteins and polyphenols content increased until $83 \mathrm{~kJ} / \mathrm{kg}$ energy inputs in the case of spearmint [61].

Xue et al. [62] showed that PEF treatment $(38.4 \mathrm{kV} / \mathrm{cm}, 272 \mu \mathrm{s})$ produced higher extraction yields of polysaccharides $(97.7 \%)$, protein $(48.9 \%)$, and polyphenolic compounds $(50.9 \%)$ in white button mushrooms compared to traditional thermal treatment $\left(95^{\circ} \mathrm{C}\right.$ for $1 \mathrm{~h}$ ). The traditional technique has a prolonged treatment cycle as compared to the PEF system. However, PEF exhibited a fluid residence time of less than $2.6 \mathrm{~min}$ [62]. In the same context, Jeya et al. [25] investigated that PEF at $7 \mathrm{kV} / \mathrm{cm}$ and $2.5 \mathrm{~kJ} / \mathrm{kg}$ resulted in a 4.2-fold enhancement in the yield of betanine as compared to untreated samples. According to the authors, $90 \%$ of betanine extraction was achieved in $35 \mathrm{~min}$. Frontuto et al. [63] indicated that the PEF pretreatment of potato peel tissues greatly enhanced the extraction of phenolics. Accordingly, PEF pretreatment achieved the same amount $(1062 \mathrm{mg} \mathrm{GAE} / \mathrm{kg})$ of phenolics in $144 \mathrm{~min}$ as compared to untreated samples (240 $\mathrm{min}$ ) [63]. Similarly, PEF treatment $(2 \mathrm{kV} / \mathrm{cm}, 11.225 \mathrm{~kJ} / \mathrm{kg})$ significantly enhanced the yield $(13.3 \%)$ of phenolic content in olive paste [64]. 
Table 1. PEF Assisted extraction of Bioactive compounds.

\begin{tabular}{|c|c|c|c|c|c|c|c|c|c|c|c|c|c|c|}
\hline Ref. & $\begin{array}{c}\text { Raw } \\
\text { Material }\end{array}$ & $\begin{array}{l}\text { Extraction } \\
\text { Technique }\end{array}$ & $\begin{array}{l}\text { Pretreatment } \\
\text { Condition }\end{array}$ & $\begin{array}{l}\text { Extraction } \\
\text { Condition }\end{array}$ & $\begin{array}{l}\text { Solid- } \\
\text { Solvent } \\
\text { Ratio }\end{array}$ & Solvent & Yield & TPC & DPPH & FRAP & IC50 & TFC & TAC & TCC \\
\hline [65] & $\begin{array}{l}\text { Cinnamon } \\
\text { (Cinnamo- } \\
\text { mum } \\
\text { verum) } \\
\text { powder }\end{array}$ & $\begin{array}{l}\text { PEF-assisted } \\
\text { extraction }\end{array}$ & $\begin{array}{c}\text { Frequency: } \\
1 \mathrm{~Hz} \\
\text { Voltage: } 2-6 \\
5.12 \mathrm{kV} / \mathrm{cm} \\
\text { No. of pulses: } \\
40-60 \mathrm{NR}\end{array}$ & $\begin{array}{l}\text { Temperature: } \\
\text { ambient } \\
\text { Time: } 48 \mathrm{~h}\end{array}$ & $1: 10 w / v$ & $\begin{array}{l}\text { Ethanol } \\
100 \mathrm{~mL}\end{array}$ & $5.06 \%$ & $\begin{array}{l}505.9 \mathrm{mg} \\
\mathrm{GA} / \mathrm{kg}\end{array}$ & $91.7 \%$ & NR & NR & NR & NR & NR \\
\hline [66] & $\begin{array}{l}\text { Nepeta bi- } \\
\text { naludensis }\end{array}$ & $\begin{array}{l}\text { PEF-assisted } \\
\text { extraction }\end{array}$ & $\begin{array}{c}\text { No. of } \\
\text { pulses: } 60 \\
\text { Frequency: } \\
1 \mathrm{~Hz} \\
\text { Voltage: } 6 \mathrm{kV} \\
\text { No. of pulses: } \\
\quad 60\end{array}$ & $\begin{array}{l}\text { Temperature: } \\
\text { ambient } \\
\text { Time: } 48 \mathrm{~h}\end{array}$ & $1: 10 w / v$ & $\begin{array}{l}\text { Ethanol } \\
100 \mathrm{~mL}\end{array}$ & $11.36 \%$ & $\begin{array}{c}417.85 \mathrm{mg} \\
\text { GA/g }\end{array}$ & $74.8 \%$ & $\begin{array}{c}1688.53 \\
\mu \mathrm{mol} \\
\mathrm{Fe}^{2+} / \mathrm{g}\end{array}$ & $\begin{array}{c}0.32 \\
\mathrm{mg} / \mathrm{mL}\end{array}$ & NR & NR & NR \\
\hline [24] & $\begin{array}{l}\text { Thinned } \\
\text { peach } \\
\text { (Prunus } \\
\text { persica) }\end{array}$ & $\begin{array}{l}\text { PEF-assisted } \\
\text { extraction }\end{array}$ & $\begin{array}{c}\text { EFS: } 0 \mathrm{kV} / \mathrm{cm} \\
\text { Specific energy: } \\
0.61-9.98 \mathrm{~kJ} / \mathrm{kg} \\
\text { Pulse } \\
\text { frequency: } \\
1 \mathrm{~Hz} \\
\text { No. of pulses } \\
\text { per time: } \\
30-150 \mu \mathrm{s}\end{array}$ & $\begin{array}{l}\text { Temperature: } \\
35^{\circ} \mathrm{C} \\
\text { Time: } 10 \mathrm{~h}\end{array}$ & NR & $\begin{array}{l}\text { Methanol } \\
80 \% \\
200 \mathrm{~mL} \\
\text { water- } \\
\text { methanol } \\
\text { solvent }\end{array}$ & NR & $\begin{array}{c}83.3 \mathrm{mg} \\
\mathrm{GAE} / 100 \mathrm{~g}\end{array}$ & $57.8 \%$ & NR & NR & $\begin{array}{c}54.3 \\
\text { CE/100 g }\end{array}$ & NR & NR \\
\hline \multirow[b]{2}{*}{ [67] } & \multirow{2}{*}{$\begin{array}{l}\text { Tomato } \\
\text { (Solanum } \\
\text { lycoper- } \\
\text { sicum) } \\
\text { peel }\end{array}$} & \multirow[b]{2}{*}{$\begin{array}{l}\text { PEF-assisted } \\
\text { extraction }\end{array}$} & $\begin{array}{c}\text { EFS: } 5 \mathrm{kV} / \mathrm{cm} \\
\text { Total specific } \\
\text { energy: } \\
5 \mathrm{~kJ} / \mathrm{kg}\end{array}$ & - & \multirow[b]{2}{*}{$1: 40 \mathrm{~g} / \mathrm{mL}$} & \multirow[b]{2}{*}{ Acetone } & \multirow[b]{2}{*}{ NR } & \multirow[b]{2}{*}{ NR } & $\begin{array}{c}4.2 \pm 0.4 \\
\mathrm{mmol} \\
\mathrm{TE} / 100 \mathrm{~g} \\
\text { FW }\end{array}$ & \multirow[b]{2}{*}{ NR } & \multirow[b]{2}{*}{ NR } & \multirow[b]{2}{*}{ NR } & \multirow[b]{2}{*}{ NR } & $\begin{array}{c}80.4 \pm 2.2 \\
\mathrm{mg} / 100 \mathrm{~g} \\
\text { FW }\end{array}$ \\
\hline & & & - & $\begin{array}{l}\text { Temperature: } \\
50 \\
\text { Time: } 4 \mathrm{~h} \\
\text { Speed: } \\
160 \mathrm{rpm}\end{array}$ & & & & & $\begin{array}{l}5.2 \pm 0.4 \\
\mathrm{mmolTE} / 100 \\
\mathrm{~g} \mathrm{FW}\end{array}$ & & & & & $\begin{array}{c}84.0 \pm 8.3 \\
\mathrm{mg} / 100 \mathrm{~g} \\
\text { FW }\end{array}$ \\
\hline
\end{tabular}


Table 1. Cont.

\begin{tabular}{|c|c|c|c|c|c|c|c|c|c|c|c|c|c|c|}
\hline Ref. & $\begin{array}{c}\text { Raw } \\
\text { Material }\end{array}$ & $\begin{array}{l}\text { Extraction } \\
\text { Technique }\end{array}$ & $\begin{array}{l}\text { Pretreatment } \\
\text { Condition }\end{array}$ & $\begin{array}{l}\text { Extraction } \\
\text { Condition }\end{array}$ & $\begin{array}{c}\text { Solid- } \\
\text { Solvent } \\
\text { Ratio }\end{array}$ & Solvent & Yield & TPC & DPPH & FRAP & IC50 & TFC & TAC & TCC \\
\hline \multirow{2}{*}{ [68] } & $\begin{array}{l}\text { Sweet } \\
\text { Cherries } \\
\text { (Prunus } \\
\text { avium) }\end{array}$ & $\begin{array}{l}\text { PEF-assisted } \\
\text { pressing }\end{array}$ & $\begin{array}{c}\text { Variable field } \\
\text { strength: } \\
1 \mathrm{kV} / \mathrm{cm} \\
\text { Frequency: } \\
5 \mathrm{~Hz} \\
\text { Pulse width: } \\
20 \mu \mathrm{s} \\
\text { Total specific } \\
\text { energy input: } \\
10 \mathrm{~kJ} / \mathrm{kg} \\
\end{array}$ & $\begin{array}{c}\text { Pressure: } \\
\text { 1.64 bar } \\
\text { Time: } \\
5 \text { min }\end{array}$ & NR & NR & $\begin{array}{c}\text { Juice } \\
\text { yield } 40 \%\end{array}$ & NR & NR & $27.4 \%$ & NR & NR & $\begin{array}{c}29.2 \pm 1.1 \\
\mathrm{mg} / 100 \mathrm{~mL}\end{array}$ & NR \\
\hline & $\begin{array}{l}\text { Sweet } \\
\text { cherries } \\
\text { (Prunus } \\
\text { avium) } \\
\text { press cake }\end{array}$ & $\begin{array}{l}\text { PEF-assisted } \\
\text { extraction }\end{array}$ & $\begin{array}{l}\text { Variable field } \\
\text { strength: } \\
0.5-1 \mathrm{kV} / \mathrm{cm} \\
\text { Frequency: } \\
5 \mathrm{~Hz} \\
\text { Pulse width: } \\
20 \mu \mathrm{s} \\
\text { Total specific } \\
\text { energy input: } \\
10 \mathrm{~kJ} / \mathrm{kg}\end{array}$ & $\begin{array}{l}\text { Time: } 24 \mathrm{~h} \\
\text { Temperature: } \\
25^{\circ} \mathrm{C}\end{array}$ & $\begin{array}{c}5: 1 \mathrm{~mL} / \mathrm{g} \\
\text { Solvent- } \\
\text { cake } \\
\text { ratio }\end{array}$ & $\begin{array}{c}\text { Acidified } \\
\text { aqueous } \\
\text { ethanol } \\
(50 \% \\
\text { ethanol; } \\
0.5 \% \mathrm{HCl} \text {, } \\
v / v)\end{array}$ & NR & NR & NR & $21.0 \%$ & NR & NR & $\begin{array}{l}218.0 \pm 14.8 \\
\mathrm{mg} / 100 \mathrm{~mL}\end{array}$ & NR \\
\hline [15] & $\begin{array}{l}\text { Moringa } \\
\text { olifera dry } \\
\text { leaves }\end{array}$ & $\begin{array}{l}\text { PEF-assisted } \\
\text { extraction }\end{array}$ & EFS: $7 \mathrm{kV} / \mathrm{cm}$ & $\begin{array}{c}\text { Time: } \\
40 \text { min } \\
\text { Temperature: } \\
\text { ambient } \\
\text { Pulse } \\
\text { duration: } \\
20 \mathrm{~ms} \\
\text { Pulse } \\
\text { interval: } \\
100 \mu \mathrm{s}\end{array}$ & NR & NR & NR & $\begin{array}{l}40.24 \mathrm{mg} \\
\text { GAE/g of } \\
\text { dry matter }\end{array}$ & 98.31 & $\begin{array}{c}108.22 \\
\mu \mathrm{moL} \\
\mathrm{AAE} / \mathrm{g} \\
\text { dry matter }\end{array}$ & NR & NR & NR & NR \\
\hline
\end{tabular}


Table 1. Cont

\begin{tabular}{|c|c|c|c|c|c|c|c|c|c|c|c|c|c|c|}
\hline Ref. & $\begin{array}{c}\text { Raw } \\
\text { Material }\end{array}$ & $\begin{array}{l}\text { Extraction } \\
\text { Technique }\end{array}$ & $\begin{array}{l}\text { Pretreatment } \\
\text { Condition }\end{array}$ & $\begin{array}{l}\text { Extraction } \\
\text { Condition }\end{array}$ & $\begin{array}{l}\text { Solid- } \\
\text { Solvent } \\
\text { Ratio }\end{array}$ & Solvent & Yield & TPC & DPPH & FRAP & IC50 & TFC & TAC & TCC \\
\hline [63] & $\begin{array}{c}\text { Potato } \\
\text { (Solanum } \\
\text { tuberosum) } \\
\text { peels }\end{array}$ & $\begin{array}{l}\text { PEF-assisted } \\
\text { extraction }\end{array}$ & $\begin{array}{c}\text { Pulse } \\
\text { width:3-25 } \mu \mathrm{s} \\
\text { Frequency: } \\
1-450 \mathrm{~Hz} \\
\text { Electric field: } \\
1 \mathrm{kV} / \mathrm{cmSpecific} \\
\text { energy: } \\
5 \mathrm{~kJ} / \mathrm{kg}\end{array}$ & $\begin{array}{l}\text { Time: } \\
\text { 30-240 min } \\
\text { Temperature: } \\
\text { 20-50 }{ }^{\circ} \mathrm{C} \\
\text { Speed: } \\
160 \mathrm{rpm}\end{array}$ & $1: 20 \mathrm{~g} / \mathrm{mL}$ & $\begin{array}{l}\text { Water- } \\
\text { ethanol } \\
\text { mixture } \\
\text { Ethanol } \\
\text { concen- } \\
\text { tration } \\
50 \%\end{array}$ & NR & $\begin{array}{c}1263.5 \pm \\
43 \mathrm{mg}- \\
\text { GAE/kg } \\
\text { FW PP }\end{array}$ & $\begin{array}{l}877.17 / \mathrm{kg} \\
\text { FWPP }\end{array}$ & NR & NR & NR & NR & NR \\
\hline [69] & $\begin{array}{c}\text { Date palm } \\
\text { (Phoenix } \\
\text { dactylifera) }\end{array}$ & $\begin{array}{l}\text { PEF-assisted } \\
\text { ethanolic } \\
\text { extraction }\end{array}$ & $\begin{array}{l}\text { EFS: } 3 \mathrm{kV} / \mathrm{cm} \\
\text { Frequenc: } 10 \mathrm{~Hz}\end{array}$ & Time: $6 \mathrm{~h}$ & $4: 1 v / v$ & $\begin{array}{c}\text { Ethanol- } \\
\text { water } \\
300 \mathrm{~mL}\end{array}$ & NR & $\begin{array}{c}67.35 \mathrm{mg} \\
\mathrm{GAE} / 100 \mathrm{~g}\end{array}$ & $50-72$ & NR & $\begin{array}{c}110 \\
\mu \mathrm{L} / \mathrm{mL}\end{array}$ & $\begin{array}{l}6.75 \mathrm{mg} \\
\mathrm{CE} / 100 \mathrm{~g}\end{array}$ & $2.08 \mathrm{mg} / \mathrm{L}$ & $\begin{array}{c}6.10 \\
\mu \mathrm{g} / \mathrm{mL}\end{array}$ \\
\hline [70] & $\begin{array}{l}\text { Tropical } \\
\text { almond } \\
\text { red leaves } \\
\text { (Terminalia } \\
\text { catappa) }\end{array}$ & $\begin{array}{l}\text { PEF-assisted } \\
\text { extraction }\end{array}$ & NR & $\begin{array}{c}\text { Frequency: } \\
1 \mathrm{~Hz} \\
\text { Electric } \\
\text { field } \\
\text { intensity: } \\
0.75 \mathrm{kV} / \mathrm{cm} \\
\text { No. of } \\
\text { pulses: } \\
50 \mathrm{n}\end{array}$ & $1: 10$ & Water & $74.6 \%$ & $\begin{array}{l}241.40 \pm \\
2.15 \mathrm{mg} \\
\mathrm{GAE} / \mathrm{g}\end{array}$ & $\begin{array}{c}93.40 \pm \\
1.23 \%\end{array}$ & NR & $\begin{array}{c}42 \\
\mathrm{mg} / \mathrm{mL}\end{array}$ & NR & NR & NR \\
\hline [59] & $\begin{array}{l}\text { Red onion } \\
\text { (Allium } \\
\text { cepa) }\end{array}$ & $\begin{array}{c}\text { PEF-assisted } \\
\text { water } \\
\text { extraction }\end{array}$ & $\begin{array}{c}\text { Pulse } \\
\text { wide: } 100 \mu \mathrm{s} \\
\text { Frequency: } \\
1 \mathrm{~Hz} \\
\text { Electric field } \\
\text { intensity: } \\
2.5 \mathrm{kV} / \mathrm{cm} \\
\text { No. of pulse: } \\
90 \\
\text { Specific energy: } \\
0.23-9.38 \mathrm{~kJ} / \mathrm{kg}\end{array}$ & $\begin{array}{l}\text { Time: } 2 \mathrm{~h} \\
\text { Shaking } \\
\text { speed: } \\
200 \mathrm{rpm} / \mathrm{min} \\
\text { Temperature: } \\
42.5^{\circ} \mathrm{C}\end{array}$ & NR & $\begin{array}{c}\text { Distilled } \\
\text { water } \\
50 \mathrm{~mL}\end{array}$ & NR & $\begin{array}{c}102.86 \mathrm{mg} \\
\mathrm{GAE} / 100 \\
\mathrm{~g} \mathrm{FW}\end{array}$ & $262.39 \%$ & NR & NR & $\begin{array}{c}37.58 \mathrm{mg} \\
\mathrm{QE} / 100 \\
\mathrm{gFW}\end{array}$ & NR & NR \\
\hline
\end{tabular}


Table 1. Cont

\begin{tabular}{|c|c|c|c|c|c|c|c|c|c|c|c|c|c|c|}
\hline Ref. & $\begin{array}{c}\text { Raw } \\
\text { Material }\end{array}$ & $\begin{array}{l}\text { Extraction } \\
\text { Technique }\end{array}$ & $\begin{array}{l}\text { Pretreatment } \\
\text { Condition }\end{array}$ & $\begin{array}{l}\text { Extraction } \\
\text { Condition }\end{array}$ & $\begin{array}{l}\text { Solid- } \\
\text { Solvent } \\
\text { Ratio }\end{array}$ & Solvent & Yield & TPC & DPPH & FRAP & IC50 & TFC & TAC & TCC \\
\hline [71] & $\begin{array}{l}\text { Wild blue- } \\
\text { berries } \\
\text { (Vaccinium } \\
\text { myrtillus) }\end{array}$ & $\begin{array}{l}\text { PEF-assisted } \\
\text { pressing }\end{array}$ & $\begin{array}{c}\text { EFS:3 kV/cm } \\
\text { Total specific } \\
\text { energy: } \\
10 \mathrm{~kJ} / \mathrm{kg} \\
\text { Frequency: } \\
10 \mathrm{~Hz} \\
\text { Pulse width: } \\
20 \mu \mathrm{s}\end{array}$ & $\begin{array}{l}\text { Time: } \\
8 \text { min } \\
\text { Pressure: } \\
1.32 \text { bar }\end{array}$ & NR & NR & 56.3 & $45.5 \%$ & NR & $35.9 \%$ & NR & NR & $77.5 \%$ & NR \\
\hline [72] & $\begin{array}{l}\text { Sour } \\
\text { cherries } \\
\text { (Prunus } \\
\text { cerasus) }\end{array}$ & $\begin{array}{l}\text { PEF-assisted } \\
\text { pressing }\end{array}$ & $\begin{array}{c}\text { EFS: } 5 \mathrm{kV} / \mathrm{cm} \\
\text { total specific } \\
\text { energy } \\
\text { input: } 10 \mathrm{~kJ} / \mathrm{kg} \\
\text { constant } \\
\text { frequency: } \\
10 \mathrm{~Hz} \\
\text { pulse } \\
\text { width: } 20 \mu \mathrm{s}\end{array}$ & $\begin{array}{l}\text { Pressing } \\
\text { time: } 9 \text { min }\end{array}$ & NR & NR & $\begin{array}{c}37.7 \mathrm{~g} \\
100 / \mathrm{g} 1\end{array}$ & $\begin{array}{c}133.90 \pm \\
2.67 \\
\mathrm{mg} \\
100 / \mathrm{mL} \\
\text { Juice }\end{array}$ & NR & $\begin{array}{c}6.60 \pm 0.13 \\
\mu \mathrm{mol} \\
\mathrm{TE} / \mathrm{mL}\end{array}$ & NR & NR & $\begin{array}{c}53.30 \pm \\
0.97 \\
\mathrm{mg} \\
100 / \mathrm{mL} \\
\text { Juice }\end{array}$ & NR \\
\hline [73] & $\begin{array}{c}\text { Fresh } \\
\text { pomelo } \\
\text { fruits } \\
\text { (Shantian } \\
\text { Variety) }\end{array}$ & - & $\begin{array}{c}\text { Number of } \\
\text { pulses of } 30 \\
\text { Electric field } \\
\text { intensity: } \\
4 \mathrm{kV} / \mathrm{cm}\end{array}$ & $\begin{array}{c}\text { Temperature: } \\
40^{\circ} \mathrm{C}\end{array}$ & $40: 60, v / v$ & $\begin{array}{c}90 \mathrm{~mL} \\
\text { ethanol- } \\
\text { water }\end{array}$ & $\begin{array}{c}16.19 \\
\mathrm{mg} / \mathrm{mL} \\
\text { (naringin) } \\
\text { increased } \\
\text { by about } \\
20 \%\end{array}$ & NR & $\begin{array}{c}38.58 \% \\
\text { increased } \\
\text { by } 70 \%\end{array}$ & NR & NR & NR & NR & NR \\
\hline [74] & $\begin{array}{l}\text { Thawed } \\
\text { blueber- } \\
\text { ries }\end{array}$ & $\begin{array}{l}\text { PEF-assisted } \\
\text { pressing }\end{array}$ & $\begin{array}{c}\text { EFSs: } 1 \text {, } \\
3 \mathrm{kV} / \mathrm{cm} \\
\text { Total specific } \\
\text { energy: } \\
10 \mathrm{~kJ} / \mathrm{kg} \\
\text { Constant } \\
\text { frequency: } \\
20 \mathrm{~Hz} \\
\text { Pulse width: } 20\end{array}$ & $\begin{array}{c}\text { Constant } \\
\text { pressure: } \\
1.32 \text { bar } \\
\text { Time: } \\
8 \mathrm{~min}\end{array}$ & NR & NR & $36.3 \pm 1 \%$ & $8.0 \%$ & NR & NR & NR & NR & $8.3 \%$ & NR \\
\hline
\end{tabular}

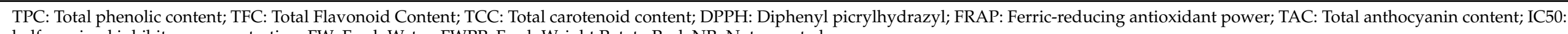
half-maximal inhibitory concentration; FW: Fresh Water; FWPP: Fresh Weight Potato Peel; NR: Not reported. 


\subsection{Agro-Industrial Waste}

Fruit and vegetable flesh or pulp is consumed while the other components, which are not mostly consumed, such as peel and seeds, contain a significant amount of various vital nutrients and phytochemicals $[9,75,76]$. For example, lemon, grapes, orange peels, avocado, jackfruit, longan, and mango seeds contain more than a $15 \%$ phenolic concentration, which is higher than those contained in fruit pulp [77]. In the food industry, much of the waste being produced is marked by the demand for biological oxygen and the demand for chemical oxygen. The food waste is rich in numerous bioactive components such as phenolic acids, flavonoids (hesperetin, quercetin, genistein, and kaempferol), and carotenoids (lutein and zeaxanthin); hence, it is concerned with the benefits of using PEF to recover valuable compounds that would contribute to the notion of zero waste.

Conventionally, these compounds are extracted by different treatments such as nanoemulsions used in the nutraceutical, cosmetics, and pharmaceutical industries [75]. The traditional solvent extraction techniques are time and energy consuming. For instance, the industrial batch extraction of polyphenols from grape peels is usually carried out at $50-60{ }^{\circ} \mathrm{C}$ for $20 \mathrm{~h}$. Furthermore, the bioactive is enclosed in plant cell vacuoles and membrane bilayers (insoluble structures) that are not open to solvents. The usage of high temperature to enhance mass transfer and reduction in time may have serious drawbacks since high temperature (greater than $70^{\circ} \mathrm{C}$ ) caused rapid degradation of heat-sensitive components such as anthocyanins [78]. Kantar et al. [79] compared the total polyphenolic content of orange, grapefruit, and lemon in both juice extract and peel (flavedo/albedo) using PEF $(3-10 \mathrm{kV} / \mathrm{cm})$ as a pretreatment followed by the traditional extraction procedure ( $50 \%$ ethanol for $1 \mathrm{~h}$ ). The results showed an improved flavonoid content and total phenolic contents (2200 mg GAE/100 g) of fruit peels at $10 \mathrm{kV} / \mathrm{cm}$ [79].

\subsection{Herbs and Spices}

Herbs and spices have been widely used to strengthen or enhance the taste and preserve the quality of food along with their beneficial effects on human health. The plant essential oils contain $85 \%$ of polyphenols, terpenes, monoterpenes, and sesquiterpenes. However, essential oils (derived from herbs and spices) have 70 or more different molecules of phytochemicals such as terpenoids, polyphenols, flavonols, flavonoids, and tannins [80-82]. Spices have been used in traditional medicine since ancient times; however, their beneficial health effects have been experimentally recognized only in the last three decades. Recently, the antioxidant properties of black pepper (piperine), red pepper (capsaicin), turmeric (curcumin), fenugreek, ginger (gingero), garlic, clove (eugenol), and onion (quercetin) have been investigated by [83]. The antioxidants derived from spices have been evaluated for the prevention of various health-related disorders including atherogenesis.

The most common method used for extracting bioactive or essential oils from plant sources is Soxhlet extraction (hydro and steam distillation), while other traditional extraction techniques include maceration, engraving, and cohobation. However, steam distillation has been used widely for the commercial production of essential oils [84]. The traditional methods are complex multistage processes, which consumed more organic solvent, time, and energy while resulting in the loss of analytes. These factors are leading to the low selectivity of conventional extraction methods. In contrast, the modern PEF technique enhanced the extraction capacity of bioactive from metabolically active tissues via increasing osmotic dehydration with less energy input and an enhanced recovery of nutrients [85]. Furthermore, PEF extraction was facilitated by solvent diffusion and freeze-drying [11].

Phyllanthus emblica L. (Syn. Emblica officinalis) is an ancient herb well known for its functional properties. PEF (18 to $24 \mathrm{kV} / \mathrm{cm}, 300$ to $1000 \mu \mathrm{s}$ ) treated Emblica juice showed an improved (9 times) extraction of quercetin and ellagic acid than thermally processed juice. According to the authors, $22 \mathrm{kV} / \mathrm{cm}$ was the optimum electric field power for 0.79 disintegration index within $500 \mu \mathrm{s}$ [85]. Similarly, PEF extraction at $20 \mathrm{kV} / \mathrm{cm}$ using $70 \%$ ethanol and water increased the yield $(12.69 \mathrm{mg} / \mathrm{g})$ of ginsenosides as compared to 
ultrasound assisted extraction, microwave assisted extraction, heat reflux extraction, and pressurized liquid extraction. The authors stated that it took less than $1 \mathrm{~s}$ to complete PEF extraction, which is much lower than the other methods tested [86].

\subsection{Leaves}

According to Segovia et al. [87], PEF treatment $(300 \mathrm{~Hz}, 30 \mathrm{kV})$ increased the polyphenols from 1.3 to $6.6 \%$ and the Oxygen Radical Absorption Capacity from 2.0 to $13.7 \%$ in Borago officinalis L. leaves. Moreover, PEF-assisted extraction increased the antioxidant capacity of the extracts and reduced the extraction times [87]. Barba et al. [88] established that PEF extraction treatments ( 0 to $141 \mathrm{~kJ} / \mathrm{kg}$, using water as a solvent) disrupt the plant cells of Stevia rebaudiana Bertoni leaves. PEF extraction improved conductivity $(\approx 25 \%)$ and soluble matter extraction yields $(\approx 33 \%)$ as compared to diffusion. Furthermore, PEF enhanced the antioxidant activity (50\%), total phenolic compounds activity $(80 \%)$, chlorogenic acid (93\%), caffeic acids (55\%), ferulic acid (90\%), and protocatechuic acids (45\%) [88]. Table 2 summarizes the range of parameters used in PEF-assisted extraction for the release of bioactive components from Agro-Industrial waste. 
Table 2. The extraction of bioactive compounds assisted by PEF pretreatment.

\begin{tabular}{|c|c|c|c|c|c|c|c|c|c|c|c|c|c|}
\hline Ref. & $\begin{array}{c}\text { Raw } \\
\text { Material }\end{array}$ & $\begin{array}{c}\text { PEF- } \\
\text { Pretreatment }\end{array}$ & $\begin{array}{l}\text { Extraction } \\
\text { Method }\end{array}$ & $\begin{array}{l}\text { Extraction } \\
\text { Conditions }\end{array}$ & $\begin{array}{c}\text { Solid- } \\
\text { Solvent } \\
\text { Ratio }\end{array}$ & Solvent & Yield & TPC & AA & TAC & DPPH & FRAP & TFC \\
\hline [89] & $\begin{array}{l}\text { Thawed } \\
\text { blackcur- } \\
\text { rant } \\
\text { (Ribes } \\
\text { nigrum) }\end{array}$ & $\begin{array}{c}\text { EFS: } 1318 \\
\text { kV/cm } \\
\text { Pulses: } 315\end{array}$ & $\begin{array}{l}\text { Cold } \\
\text { pressing }\end{array}$ & $\begin{array}{l}\text { Power: } \\
150 \mathrm{~W} \\
\text { Velocity: } \\
70 \mathrm{rpm} \\
\text { Pressing } \\
\text { time: } \\
1.5 \mathrm{~min}\end{array}$ & NR & NR & NR & $\begin{array}{c}3.8 \pm 0.2 \\
\mathrm{mg} \text { GA/g }\end{array}$ & $\begin{array}{l}1.88 \pm 0.06 \\
\mathrm{mg} \mathrm{GA} / \mathrm{g}\end{array}$ & NR & NR & NR & NR \\
\hline [90] & $\begin{array}{l}\text { Fresh tea } \\
\text { leaves } \\
\text { (Camellia } \\
\text { sinensis) }\end{array}$ & $\begin{array}{c}\text { EFS: } \\
1.00 \mathrm{kV} / \mathrm{cm} \\
\text { Pulses: } 100 \\
\text { Energy: } \\
22 \mathrm{~kJ} / \mathrm{kg} \\
\text { Temperature: } \\
1.5^{\circ} \mathrm{C}\end{array}$ & $\begin{array}{l}\text { Organic } \\
\text { solvent } \\
\text { extraction }\end{array}$ & $\begin{array}{l}\text { Time: } 2 \mathrm{~h} \\
\text { Temperature: } \\
\text { room } \\
\text { Stirring } \\
\text { speed: } \\
250 \mathrm{rpm}\end{array}$ & $\begin{array}{c}1: 1000 \\
\text { Biomass to } \\
\text { solvent ratio }\end{array}$ & $\begin{array}{c}50 \% \\
\text { acetone/water } \\
(w / w) \\
\text { solution }\end{array}$ & NR & $398 \mathrm{mg} / \mathrm{L}$ & NR & NR & NR & NR & NR \\
\hline [72] & $\begin{array}{l}\text { Sour cherry } \\
\text { (Prunus } \\
\text { cerasus) } \\
\text { press cake }\end{array}$ & $\begin{array}{c}\text { EFS: } \\
5 \mathrm{kV} / \mathrm{cm} \\
\text { Specific } \\
\text { energy } \\
\text { input: } \\
10 \mathrm{~kJ} / \mathrm{kg} \\
\text { Frequency: } \\
10 \mathrm{~Hz} \\
\text { Pulse width: } \\
20 \mu \mathrm{s}\end{array}$ & $\begin{array}{c}\text { Solvent } \\
\text { extraction }\end{array}$ & $\begin{array}{l}\text { Time: } 24 \mathrm{~h} \\
\text { Temperature: } \\
\text { ambient }\end{array}$ & $\begin{array}{c}10: 1 \\
v / w \\
\text { solvent to } \\
\text { press cake }\end{array}$ & $\begin{array}{c}\text { Acidified } \\
\text { aqueous } \\
\text { methanol } \\
70 \% \mathrm{MeOH} \\
\text { and } 0.5 \% \\
\mathrm{HCl}, v / v\end{array}$ & NR & $\begin{array}{c}407.90 \pm \\
18.54 \\
\mathrm{mg} / 100 \mathrm{~g}\end{array}$ & NR & $\begin{array}{c}168.50 \pm \\
3.99 \\
\mathrm{mg} / 100 \mathrm{~g}\end{array}$ & NR & $\begin{array}{c}56.10 \pm 1.25 \\
\mu \mathrm{mol} \mathrm{TE} / \mathrm{g}\end{array}$ & NR \\
\hline [91] & $\begin{array}{c}\text { Basil leaves } \\
\text { (Ocimum } \\
\text { Ameri- } \\
\text { canum) }\end{array}$ & $\begin{array}{c}\text { EFS: } \\
2-3 \mathrm{kV} / \mathrm{cm} \\
\text { Time: } \\
1-2 \mathrm{~min}\end{array}$ & $\begin{array}{l}\text { Conventional } \\
\text { method: } \\
\text { maceration }\end{array}$ & $\begin{array}{l}\text { Time: } 3 \mathrm{~h} \\
\text { Temperature: } \\
\text { room }\end{array}$ & NR & $\begin{array}{c}\text { Distilled } \\
\text { water } \\
300 \mathrm{~mL}\end{array}$ & $\begin{array}{c}33.15 \% \pm \\
2.484 \%\end{array}$ & $\begin{array}{c}115.203 \pm \\
1.115 \mathrm{mg} \\
\mathrm{GAE} / \mathrm{g} \\
\text { extract }\end{array}$ & NR & NR & NR & NR & $\begin{array}{c}75.816 \pm \\
0.723 \mathrm{mg} \\
\mathrm{QE} / \mathrm{g}\end{array}$ \\
\hline
\end{tabular}


Table 2. Cont.

\begin{tabular}{|c|c|c|c|c|c|c|c|c|c|c|c|c|c|}
\hline Ref. & $\begin{array}{c}\text { Raw } \\
\text { Material }\end{array}$ & PEF-Pretreatment & $\begin{array}{l}\text { Extraction } \\
\text { Method }\end{array}$ & $\begin{array}{l}\text { Extraction } \\
\text { Conditions }\end{array}$ & $\begin{array}{c}\text { Solid- } \\
\text { Solvent } \\
\text { Ratio }\end{array}$ & Solvent & Yield & TPC & AA & TAC & DPPH & FRAP & TFC \\
\hline \multirow[t]{2}{*}{ [19] } & $\begin{array}{l}\text { Rosmary } \\
\text { (Salvia } \\
\text { rosmari- } \\
\text { nusby)—product }\end{array}$ & $\begin{array}{c}\text { Frequency: } 10 \mathrm{~Hz} \\
\text { Pulse width: } 30 \mu \mathrm{s} \\
\text { Pulses: } 167 \\
\text { EFS: } 1.1 \mathrm{kV} / \mathrm{cm} \\
\text { Specific energy input } \\
0.36 / \mathrm{kg} \\
\text { ct } 24 \mathrm{~g} \text { of } 0.1 \% \text { aqueous } \\
\mathrm{NaCl}(1: 1.4 \mathrm{w} / \mathrm{v}) \\
\end{array}$ & \multirow{2}{*}{$\begin{array}{l}\text { Ultrasound } \\
\text { assisted }\end{array}$} & \multirow{2}{*}{$\begin{array}{c}\text { Power: } \\
200 \mathrm{~W} \\
\text { Temperature: } \\
40{ }^{\circ} \mathrm{C} \\
\text { Time: } \\
12.48 \mathrm{~min}\end{array}$} & \multirow[t]{2}{*}{$(1: 20 w / v)$} & \multirow{2}{*}{$\begin{array}{c}100 \mathrm{~mL} \text { of } \\
55.19 \% \\
\text { aqueous } \\
\text { EtOH }\end{array}$} & \multirow[t]{2}{*}{ NR } & $\begin{array}{c}297 \mathrm{mg} \\
\mathrm{GAE} / 100 \mathrm{~g} \\
\mathrm{FW}\end{array}$ & \multirow[t]{2}{*}{ NR } & \multirow[t]{2}{*}{ NR } & $\begin{array}{c}593 \mathrm{mg} \\
\mathrm{TE} / 100 \mathrm{~g} \\
\text { FW }\end{array}$ & \multirow[t]{2}{*}{ NR } & \multirow[t]{2}{*}{ NR } \\
\hline & $\begin{array}{c}\text { Thyme } \\
\text { (Thymus } \\
\text { vulgaris) } \\
\text { by-product }\end{array}$ & $\begin{array}{c}\text { Frequency: } 10 \mathrm{~Hz} \\
\text { Pulse width: } 30 \mu \mathrm{s} \\
\text { Pulses: } 167 \\
\text { EFS: } 1.1 \mathrm{kV} / \mathrm{cm} \\
\text { Specific energy input } \\
0.46 \mathrm{~kJ} / \mathrm{kg} \\
24 \mathrm{~g} \text { of } 0.1 \% \text { aqueous } \\
\mathrm{NaCl} 24 \mathrm{~g} \text { of } 0.1 \% \\
\text { aqueous } \mathrm{NaCl} \\
(1: 1.5 \mathrm{w} / \mathrm{v})\end{array}$ & & & & & & $\begin{array}{c}460 \mathrm{mg} \\
\mathrm{GAE} / 100 \mathrm{~g} \\
\mathrm{FW}\end{array}$ & & & $\begin{array}{c}570 \mathrm{mg} \\
\mathrm{TE} / 100 \mathrm{~g} \\
\text { FW }\end{array}$ & & \\
\hline
\end{tabular}

AA: Antioxidant activity; FRAP: Ferric-reducing antioxidant power; TPC: Total phenolic content; TPC: Total phenolic content; TFC: Total flavonoid content; EFS: Electric field strength; NR: not reported. 


\subsection{Oleaginous Seeds}

The major oil crops include ground nuts, olive, linseed, cotton, hemp, castor, cottonseed, safflower, sesame oils, etc. Among them soy, rape, palm, and sunflower are the most significant oil-bearing plants [92]. Fats and oils are the most consumed items and are of much importance because of triacylglycerols [93]. Furthermore, edible oils are a major source of energy (calories) and essential vitamins. Moreover, oilseeds are placed second after grains as food reserves [94,95]. Pressing is a conventional method to extract oil from seeds, which squeezes the oil out of solid material (having more than $30 \%$ oil). After pressing, the press-cake is then subjected to solvent extraction. Traditionally, cold and hot-pressing technologies are used for the extraction of oil such as solvent extraction (Soxhlet) for flaxseed. On an industrial scale, generally, the solvent extraction step is applied by using a lot of hexane in the countercurrent extractors [96]. In contrast, PEF treatments $(50 \mathrm{kV})$ were used to extract oil (including tocopherols, antioxidants, herbal cholesterol (phytosterols), and other functional compounds) from oleaginous material [15]. For instance, PEF $(2 \mathrm{kV} / \mathrm{cm}, 11.25 \mathrm{~kJ} / \mathrm{kg}, 25 \mathrm{~Hz})$ using monopolar exponential decay pulses $(0.3 \mu \mathrm{s})$ increased the oil yield $(22.66 \mathrm{~kg} / 100 \mathrm{~kg})$ from olive paste as compared to the control sample $(20.00 \mathrm{~kg} / 100 \mathrm{~kg})$ [97]. Table 3 illustrates that PEF-assisted extraction is a progressing nonthermal technique to increase the extraction yield of seed oils. PEF-assisted extraction assures higher extraction yields without a detrimental impact on the nutritional value and freshness of the product [98]. 
Table 3. PEF assisted extraction of oils.

\begin{tabular}{|c|c|c|c|c|c|c|c|}
\hline Ref. & Raw Material & $\begin{array}{c}\text { PEF Pretreatment } \\
\text { Conditions }\end{array}$ & Extraction Method & $\begin{array}{c}\text { Extraction } \\
\text { Equipment }\end{array}$ & Extraction Conditions & Solvent & Oil Yield \\
\hline [99] & Sunflower seeds & $\begin{array}{c}\text { EFS: } 7.0 \mathrm{kV} / \mathrm{cm} \\
\text { Frequency: } 0.5 \mathrm{~Hz} \\
\text { Solvent content: } 50 \mathrm{wt} . \% \\
\text { Time: } 90 \mathrm{~s} \\
\text { Pulse width: } 30 \mu \mathrm{s}\end{array}$ & Solvent extraction & Shaker & $\begin{array}{c}\text { Frequency: } 400 / \mathrm{min} \\
\text { Time: } 3 \text { h Temperature: } \\
\text { room }\end{array}$ & Bioethanol & $55.9 \%$ \\
\hline [100] & Damask rose flowers & $\begin{array}{l}\text { EFS: } 20 \mathrm{kV} / \mathrm{cm} \\
\text { Pulse number: } 8\end{array}$ & $\begin{array}{l}\text { PEF-assisted Hydro } \\
\text { distillation }\end{array}$ & $\begin{array}{l}\text { Clevenger-type } \\
\text { micro-apparatus }\end{array}$ & $\begin{array}{c}\text { Distillation temperature: } \\
100{ }^{\circ} \mathrm{C} \\
\text { Distillation time: } 2 \mathrm{~h}\end{array}$ & $10 \%$ sodium chloride & $\begin{array}{c}\text { yield of } 0.105 \% \text { with a } \\
50 \% \text { increase in } \\
\text { essential oil }\end{array}$ \\
\hline [101] & Sunflower seeds & $\begin{array}{c}\text { EFS: } 7 \mathrm{kV} / \mathrm{cm} \\
\text { Frequency: } 1.5 \mathrm{~Hz} \\
\text { Treatment time: } 30 \mathrm{~s} \\
\text { Pulse width: } 30 \mu \mathrm{s}\end{array}$ & Solvent extraction & Shaker & $\begin{array}{c}\text { Frequency: } 400 / \mathrm{min} \\
\text { Time: } 3 \mathrm{~h} \\
\text { Temperature: room }\end{array}$ & $\begin{array}{l}\text { Hexane } 40 \mathrm{~mL} \\
\quad(50 \mathrm{wt} . \%)\end{array}$ & $48.24 \%$ \\
\hline$[64]$ & $\begin{array}{c}\text { Olive fruits (Arroniz } \\
\text { variety) }\end{array}$ & NR & $\begin{array}{l}\text { Pilot PEF-assisted } \\
\text { extraction }\end{array}$ & Pilot PEF system & $\begin{array}{c}\text { Electric fields: } 2 \mathrm{kV} / \mathrm{cm} \\
\text { Frequency: } 25 \mathrm{~Hz} \\
\text { flow rate: } 520 \mathrm{~kg} / \mathrm{h} \\
\text { Specific energy: } \\
11.25 \mathrm{~kJ} / \mathrm{kg}\end{array}$ & NR & $22.66 \mathrm{~kg} / 100 \mathrm{~kg}$ \\
\hline [102] & $\begin{array}{l}\text { Olives (Nocellara del } \\
\text { Belice variety) }\end{array}$ & $\begin{array}{c}\text { EFS: } 2 \mathrm{kV} / \mathrm{cm} \\
\text { Frequency: } 25 \mathrm{~Hz} \\
\text { Pulse width: } 50 \mu \mathrm{s} \\
\text { Mass flow rate: } \\
2300 \mathrm{~kg} / \mathrm{h} \\
\text { Energy delivered per } \\
\text { pulse: } 210 \mathrm{~J} \\
\text { Specific energy: } \\
7.83 \mathrm{~kJ} / \mathrm{kg}\end{array}$ & NR & $\begin{array}{l}\text { Hammer crusher } \\
\text { Malaxation machine }\end{array}$ & $\begin{array}{l}\text { Malaxation time: } 30 \mathrm{~min} \\
\text { Temperature: } 27^{\circ} \mathrm{C}\end{array}$ & NR & $85.5 \%$ \\
\hline
\end{tabular}




\subsection{Microorganisms}

Microorganisms such as bacteria, yeast, and algae are good sources for highly valued compounds such as enzymes, pigments, and nutrients as shown in Table 4. The major portion of such compounds resides inside the cell; therefore, it is essential to extract and purify them before use [103]. Microorganisms can carry out a broad range of reactions and are adaptable to a variety of conditions. They can be moved from nature into the laboratory to produce beneficial compounds on cheap sources such as carbon and nitrogen. Due to the biological activity of secondary metabolites originating from microbes, they are greatly beneficial for our nutrition and health. Research is progressed in the screening of naturally occurring microbial products, for the development of novel therapeutic agents. The search of novel chemicals is an important way forward to study the capacity of lesser-known or novel bacterial taxa [104].

Martínez et al. [105] used PEF (15-25 kV/ cm, 60-150 $\left.\mu \mathrm{s}, 10-40{ }^{\circ} \mathrm{C}\right)$ to enhance the extraction of a selective protein called phycocyanin (a water-soluble protein) from Artrosphira platensisto's fresh biomass. According to the authors, low molecular weight compounds move directly through the cytoplasmic membrane after electroporation, while high molecular weight compounds require that the pores produced by the PEF treatment expand over time. Hence, the resultant delay of $150 \mathrm{~min}$ was recorded during the extraction. [105]. Similarly, Jaeschke et al. [106] also succeeded in achieving a high protein and phycocyanin yield from Arthospira platensis after a PEF treatment of $40 \mathrm{kV} / \mathrm{cm}$ utilizing pulses of $1 \mu \mathrm{s}(112 \mathrm{~kJ} / \mathrm{kg})$ [106]. Martínez et al. [107] extracted another water-soluble protein ( $\beta$-phycoerythrin) from Porphyridium cruentum by applying PEF for $24 \mathrm{~h}$. However, this water-soluble phycobiliprotein was unobservable in the untreated cells, even after extended periods of incubation. The results showed that the extraction of $\beta$-phycoerythrin involved not only the diffusion of the pigment through the cell membrane, but also the disassembly from the cell organization of the molecule. In this way, PEF released the P. cruentum organelles (hydrolytic enzymes) that broke the bonds between the pigment and other cell compounds; thus, the water- $\beta$-phycoerythrin complex diffused, carried by a concentration gradient, through the membrane. The enzymatic autolysis of microalgae caused by PEF must be further investigated for the industrial implementation of PEF extraction technology [108]. Table 4 further confines some of the literature on PEF that involves the extraction of other phytoconstituents.

Table 4. The extraction of other phytoconstituents.

\begin{tabular}{|c|c|c|c|c|c|c|}
\hline \multicolumn{7}{|c|}{ Phycocyanin } \\
\hline References & Raw material & PEF treatment & $\begin{array}{l}\text { Extraction } \\
\text { method }\end{array}$ & $\begin{array}{l}\text { Extraction } \\
\text { conditions }\end{array}$ & Solvent & Yield \\
\hline [105] & $\begin{array}{l}\text { Arthrospira } \\
\text { platensis }\end{array}$ & $\begin{array}{c}\text { EFS: } 25 \mathrm{kV} / \mathrm{cm} \\
\text { Treatment time: } \\
150 \mu \mathrm{s} \\
\text { Temperature: } \\
40^{\circ} \mathrm{C}\end{array}$ & $\begin{array}{l}\text { PEF-assisted } \\
\text { extraction }\end{array}$ & $\begin{array}{c}\text { Temperature: } \\
20^{\circ} \mathrm{C} \\
\text { Time: } 360 \mathrm{~min}\end{array}$ & $\begin{array}{c}19 \mathrm{~mL} \text { of distilled } \\
\text { water }\end{array}$ & $\begin{array}{l}\text { Extraction } \\
\text { yield } \\
151.94 \pm \\
14.22 \mathrm{mg} / \mathrm{g}\end{array}$ \\
\hline \multicolumn{7}{|c|}{ Total Amino Nitrogen Content (TANC) } \\
\hline References & Raw material & PEF treatment & $\begin{array}{l}\text { Extraction } \\
\text { method }\end{array}$ & $\begin{array}{l}\text { Extraction } \\
\text { conditions }\end{array}$ & Solvent & Yield \\
\hline [43] & $\begin{array}{c}\text { Fresh abalone } \\
\text { (Haliotis Discus } \\
\text { Hannai Ino) } \\
\text { viscera }\end{array}$ & NR & $\begin{array}{l}\text { PEF-assisted } \\
\text { enzymatic } \\
\text { extraction }\end{array}$ & $\begin{array}{c}\text { EFS: } 20 \mathrm{kV} / \mathrm{cm} \\
\text { treatment times: } \\
600 \mathrm{~s}\end{array}$ & NR & $\begin{array}{c}42.35 \% \\
175.20 \mathrm{mg} / 100 \mathrm{~mL}\end{array}$ \\
\hline
\end{tabular}


Table 4. Cont.

\begin{tabular}{|c|c|c|c|c|c|c|}
\hline \multicolumn{7}{|c|}{ Lipids } \\
\hline References & Raw material & PEF treatment & $\begin{array}{l}\text { Extraction } \\
\text { method }\end{array}$ & $\begin{array}{l}\text { Extraction } \\
\text { conditions }\end{array}$ & Solvent & Extracted lipid \\
\hline [109] & $\begin{array}{l}\text { fresh microalgae } \\
\text { Auxenochlorella } \\
\text { protothecoides }\end{array}$ & $\begin{array}{c}\text { Flow rate: } \\
0.1 \mathrm{~mL} / \mathrm{s} \\
\text { Pulse duration } \\
1 \mu \mathrm{s} \\
\text { EFS: } 4 \mathrm{MV} / \mathrm{m} \\
\text { Specific energy: } \\
150 \mathrm{~kJ} / \mathrm{L}\end{array}$ & NR & $\begin{array}{l}\text { Time: overnight } \\
\text { incubation } \\
\text { conditions: } \\
\text { Temperature: } \\
25^{\circ} \mathrm{C} \\
\text { Time: } 20 \mathrm{~h}\end{array}$ & $\begin{array}{l}\text { Hexane-ethanol } \\
\text { blend }\end{array}$ & $97 \%$ \\
\hline \multicolumn{7}{|c|}{ Pectin } \\
\hline References & Raw material & $\begin{array}{l}\text { extraction } \\
\text { method }\end{array}$ & Extraction device & $\begin{array}{l}\text { Extraction } \\
\text { conditions }\end{array}$ & $\begin{array}{l}\text { Powder-solvent } \\
\text { ratio }\end{array}$ & Yield \\
\hline$[110]$ & $\begin{array}{l}\text { Raw jackfruit } \\
\text { (Artocarpus } \\
\text { heterophyllus) }\end{array}$ & $\begin{array}{l}\text { Combination of } \\
\text { PEF- and } \\
\text { microwave- } \\
\text { assisted } \\
\text { extraction }\end{array}$ & $\begin{array}{l}\text { PEF generator } \\
\text { microwave } \\
\text { reactor }\end{array}$ & $\begin{array}{c}\text { PEF conditions: } \\
\text { Time: } 5 \text { min, EFS: } \\
10 \mathrm{kV} / \mathrm{cm} . \\
\text { Microwave } \\
\text { conditions: Time: } \\
10 \text { min, Power } \\
\text { level of } 650 \mathrm{~W} / \mathrm{g}\end{array}$ & $1: 4$ & $18.3 \%$ \\
\hline \multicolumn{7}{|c|}{ Cellulose } \\
\hline References & Raw material & PEF treatment & $\begin{array}{l}\text { Extraction } \\
\text { method }\end{array}$ & Solvent & \multicolumn{2}{|c|}{ Yield } \\
\hline [39] & $\begin{array}{l}\text { Mendong Fiber } \\
\text { (Fimbristylis } \\
\text { globulosa) }\end{array}$ & $\begin{array}{c}\text { EFS: } 1.3 \mathrm{kV} / \mathrm{cm} \\
\text { Frequency: } \\
20 \mathrm{kHz} \\
\text { Time } 30 \mathrm{~s}\end{array}$ & $\begin{array}{l}\text { PEF-assisted } \\
\text { alkali extraction }\end{array}$ & $\begin{array}{l}\mathrm{NaOH} \text { sol. } \\
(300 \mathrm{~mL}) \\
60 \% \text { conc. }\end{array}$ & \multicolumn{2}{|c|}{$97.8 \%$} \\
\hline \multicolumn{7}{|c|}{ Pigment } \\
\hline References & Raw material & PEF treatment & $\begin{array}{l}\text { Extraction } \\
\text { method }\end{array}$ & $\begin{array}{l}\text { Extraction } \\
\text { conditions }\end{array}$ & \multicolumn{2}{|c|}{ Yield } \\
\hline$[111]$ & $\begin{array}{l}\text { Algae paste of } \\
\text { Nannochloropsis } \\
\text { oceanica }\end{array}$ & $\begin{array}{l}\text { Constant flow } \\
\text { rate: } 20 \mathrm{~mL} / \mathrm{min} \\
\text { Temperature: } \\
25^{\circ} \mathrm{C} \\
\text { Square wave: } 5 \mu \mathrm{s} \\
\text { EFS: } 10 \mathrm{kV} / \mathrm{cm} \\
\text { Total specific } \\
\text { energy } \\
\text { inputs: } 100 \mathrm{~kJ} / \mathrm{kg}\end{array}$ & $\begin{array}{c}\text { PEF-assisted } \\
\text { supercritical } \mathrm{CO}_{2} \\
\text { extraction }\end{array}$ & $\begin{array}{c}\text { Pressure: } 8,14, \\
\text { and } 20 \mathrm{MPa} \\
\text { Fixed } \\
\text { temperature: } \\
35^{\circ} \mathrm{C} \\
\mathrm{CO}_{2} / \text { biomass } \\
\text { ratio: } \\
53.3 \mathrm{kgCO} / \mathrm{kg} \\
\mathrm{DW} \\
\text { Holding time: } \\
7 \text { min }\end{array}$ & \multicolumn{2}{|c|}{$\begin{array}{l}\text { Total carotenes: } 36 \% \\
\text { Total chlorophyll: 52\% }\end{array}$} \\
\hline
\end{tabular}

EFS: Electric field strength; NR: not reported.

\section{Conclusions}

PEF uses a moderate to high electric field, keeping low energy, low solvent, and less time along with higher extraction yields of different bioactive compounds from fruits, vegetables, herbs, spices, leaves, and their wastes. Furthermore, PEF-assisted extraction enhanced the oil release from oleaginous seeds in comparison to other conventional techniques. Industrially, PEF continuous extraction technology showed promising results in the extraction rate of phytoconstituents. The efficacy of the PEF extracting system depends not only on the processing parameters but also on the solvent nature and sample composition. The targeted physicochemical properties include size, shape, $\mathrm{pH}$, conductivity, etc. However, the extracted component's size and position in the cell cytoplasm or vacuoles are also important factors. It is worthy to note that PEF exhibited potential yields of highly valued items such as enzymes, pigments, and nutrients from microorganisms such as bacteria, yeast, and algae. In future, PEF processing is a promising method for pharmaceuticals, food processing, and bioengineering industries due to its energy-efficient nature. 
Author Contributions: Conceptualization, M.M.A.N.R. and R.M.A.; resources, R.K. and B.S.; writing—original draft preparation, M.M.A.N.R., P.Ł.K., R.N.A., S.I., M.I., M.Z.K. and U.R.; writingreview and editing, M.K., M.M.A.N.R. and R.M.A.; visualization, M.K. and P.Ł.K.; supervision, R.M.A.; project administration, R.M.A. All authors have read and agreed to the published version of the manuscript.

Funding: This research received no external funding.

Institutional Review Board Statement: Not applicable.

Informed Consent Statement: Not applicable.

Data Availability Statement: Not applicable.

Acknowledgments: The authors are thankful to the University of Sargodha, Sargodha, Pakistan for their support. Rana Muhammad Aadil is thankful to the University of Agriculture, Faisalabad, Pakistan for their support.

Conflicts of Interest: The authors declare no conflict of interest.

\section{References}

1. Yammine, S.; Brianceau, S.; Manteau, S.; Turk, M.; Ghidossi, R.; Vorobiev, E.; Mietton-Peuchot, M. Extraction and purification of high added value compounds from by-products of the winemaking chain using alternative/nonconventional processes/technologies. Crit. Rev. Food Sci. Nutr. 2018, 58, 1375-1390. [CrossRef]

2. Ranjha, M.M.A.N.; Irfan, S.; Nadeem, M.; Mahmood, S. A Comprehensive Review on Nutritional Value, Medicinal Uses, and Processing of Banana. Food Rev. Int. 2020. [CrossRef]

3. Ranjha, M.M.A.N.; Shafique, B.; Wang, L.; Irfan, S.; Safdar, M.N.; Murtaza, M.A.; Nadeem, M.; Mahmood, S.; Mueen-ud-Din, G.; Nadeem, H.R. A comprehensive review on phytochemistry, bioactivity and medicinal value of bioactive compounds of pomegranate (Punica granatum). Adv. Tradit. Med. 2021. [CrossRef]

4. Nadeem, H.R.; Akhtar, S.; Ismail, T.; Sestili, P.; Lorenzo, J.M.; Ranjha, M.M.A.N.; Jooste, L.; Hano, C.; Aadil, R.M. Heterocyclic Aromatic Amines in Meat: Formation, Isolation, Risk Assessment, and Inhibitory Effect of Plant Extracts. Foods 2021, $10,1466$. [CrossRef]

5. Ali Nawaz Ranjha, M.M. A Critical Review on Presence of Polyphenols in Commercial Varieties of Apple Peel, their Extraction and Health Benefits. Open Access J. Biog. Sci. Res. 2020, 6. [CrossRef]

6. Mahmood, S. A Narrative Review on the Phytochemistry, Nutritional Profile and Properties of Prickly Pear Fruit. Open Access J. Biog. Sci. Res. 2021, 7. [CrossRef]

7. Chemat, F.; Abert Vian, M.; Fabiano-Tixier, A.S.; Nutrizio, M.; Režek Jambrak, A.; Munekata, P.E.S.; Lorenzo, J.M.; Barba, F.J.; Binello, A.; Cravotto, G. A review of sustainable and intensified techniques for extraction of food and natural products. Green Chem. 2020, 22, 2325-2353. [CrossRef]

8. Yan, L.G.; He, L.; Xi, J. High intensity pulsed electric field as an innovative technique for extraction of bioactive compounds-A review. Crit. Rev. Food Sci. Nutr. 2017, 57, 2877-2888. [CrossRef]

9. Ranjha, M.M.A.N.; Amjad, S.; Ashraf, S.; Khawar, L.; Safdar, M.N.; Jabbar, S.; Nadeem, M.; Mahmood, S.; Murtaza, M.A. Extraction of Polyphenols from Apple and Pomegranate Peels Employing Different Extraction Techniques for the Development of Functional Date Bars. Int. J. Fruit Sci. 2020, 20, S1201-S1221. [CrossRef]

10. Bansal, V.; Sharma, A.; Ghanshyam, C.; Singla, M.L.; Kim, K.H. Influence of pulsed electric field and heat treatment on Emblica officinalis juice inoculated with Zygosaccharomyces bailii. Food Bioprod. Process. 2015, 95, 146-154. [CrossRef]

11. Gabrić, D.; Barba, F.; Roohinejad, S.; Gharibzahedi, S.M.T.; Radojčin, M.; Putnik, P.; Bursać Kovačević, D. Pulsed electric fields as an alternative to thermal processing for preservation of nutritive and physicochemical properties of beverages: A review. J. Food Process. Eng. 2018, 41, 12638. [CrossRef]

12. Mohamad, A.; Shah, N.N.A.K.; Sulaiman, A.; Mohd Adzahan, N.; Aadil, R.M. Impact of the pulsed electric field on physicochemical properties, fatty acid profiling, and metal migration of goat milk. J. Food Process. Preserv. 2020, 44, 14940. [CrossRef]

13. Aadil, R.M.; Zeng, X.A.; Sun, D.W.; Wang, M.S.; Liu, Z.W.; Zhang, Z.H. Combined effects of sonication and pulsed electric field on selected quality parameters of grapefruit juice. LWT Food Sci. Technol. 2015, 62, 890-893. [CrossRef]

14. Sánchez-Vega, R.; Elez-Martínez, P.; Martín-Belloso, O. Influence of high-intensity pulsed electric field processing parameters on antioxidant compounds of broccoli juice. Innov. Food Sci. Emerg. Technol. 2015, 29, 70-77. [CrossRef]

15. Bozinou, E.; Karageorgou, I.; Batra, G.; GDourtoglou, V.; ILalas, S. Pulsed Electric Field Extraction and Antioxidant Activity Determination of Moringa oleifera Dry Leaves: A Comparative Study with Other Extraction Techniques. Beverages 2019, 5, 8. [CrossRef]

16. Evrendilek, G.A. Change regime of aroma active compounds in response to pulsed electric field treatment time, sour cherry juice apricot and peach nectars, and physical and sensory properties. Innov. Food Sci. Emerg. Technol. 2016, 33, 195-205. [CrossRef] 
17. Arshad, R.N.; Abdul-Malek, Z.; Munir, A.; Buntat, Z.; Ahmad, M.H.; Jusoh, Y.M.M.; Bekhit, A.E.D.; Roobab, U.; Manzoor, M.F.; Aadil, R.M. Electrical systems for pulsed electric field applications in the food industry: An engineering perspective. Trends Food Sci. Technol. 2020, 104, 1-13. [CrossRef]

18. López-Gámez, G.; Elez-Martínez, P.; Martín-Belloso, O.; Soliva-Fortuny, R. Changes of carotenoid content in carrots after application of pulsed electric field treatments. Lwt 2021, 147, 111408. [CrossRef]

19. Tzima, K.; Brunton, N.P.; Lyng, J.G.; Frontuto, D.; Rai, D.K. The effect of Pulsed Electric Field as a pre-treatment step in Ultrasound Assisted Extraction of phenolic compounds from fresh rosemary and thyme by-products. Innov. Food Sci. Emerg. Technol. 2021, 69, 102644. [CrossRef]

20. Soquetta, M.B.; Terra, L.D.M.; Bastos, C.P. Green technologies for the extraction of bioactive compounds in fruits and vegetables. CYTA J. Food 2018, 16, 400-412. [CrossRef]

21. Oroian, M.; Escriche, I. Antioxidants: Characterization, natural sources, extraction and analysis. Food Res. Int. 2015, 74, 10-36. [CrossRef] [PubMed]

22. Xi, J.; Li, Z.; Fan, Y. Recent advances in continuous extraction of bioactive ingredients from food-processing wastes by pulsed electric fields. Crit. Rev. Food Sci. Nutr. 2021, 61, 1738-1750. [CrossRef]

23. Panja, P. Green extraction methods of food polyphenols from vegetable materials. Curr. Opin. Food Sci. 2018, $23,173-182$. [CrossRef]

24. Redondo, D.; Venturini, M.E.; Luengo, E.; Raso, J.; Arias, E. Pulsed electric fields as a green technology for the extraction of bioactive compounds from thinned peach by-products. Innov. Food Sci. Emerg. Technol. 2018, 45, 335-343. [CrossRef]

25. Thulasidas, J.S.; Varadarajan, G.S.; Sundararajan, R. Pulsed Electric Field for Enhanced Extraction of Intracellular Bioactive Compounds from Plant Products: An Overview. Nov. Approaches Drug Des. Dev. 2019, 5. [CrossRef]

26. Koubaa, M.; Roselló-Soto, E.; Šic Žlabur, J.; Režek Jambrak, A.; Brnčić, M.; Grimi, N.; Boussetta, N.; Barba, F.J. Current and New Insights in the Sustainable and Green Recovery of Nutritionally Valuable Compounds from Stevia rebaudiana Bertoni. J. Agric. Food Chem. 2015, 63, 6835-6846. [CrossRef]

27. Barba, F.J.; Boussetta, N.; Vorobiev, E. Emerging technologies for the recovery of isothiocyanates, protein and phenolic compounds from rapeseed and rapeseed press-cake: Effect of high voltage electrical discharges. Innov. Food Sci. Emerg. Technol. 2015, 31, 67-72. [CrossRef]

28. Puértolas, E.; Koubaa, M.; Barba, F.J. An overview of the impact of electrotechnologies for the recovery of oil and high-value compounds from vegetable oil industry: Energy and economic cost implications. Food Res. Int. 2016, 80, 19-26. [CrossRef]

29. Sarkis, J.R.; Boussetta, N.; Tessaro, I.C.; Marczak, L.D.F.; Vorobiev, E. Application of pulsed electric fields and high voltage electrical discharges for oil extraction from sesame seeds. J. Food Eng. 2015, 153, 20-27. [CrossRef]

30. Barba, F.J.; Parniakov, O.; Koubaa, M.; Lebovka, N. Pulsed Electric Fields Assisted Extraction from Exotic Fruit Residues. In Handbook of Electroporation; Miklavcic, D., Ed.; Springer: Cham, Switzerland, 2016; pp. 1-18. ISBN 978-3-319-26779-1.

31. Teh, S.S.; Niven, B.E.; Bekhit, A.E.D.A.; Carne, A.; Birch, E.J. Microwave and pulsed electric field assisted extractions of polyphenols from defatted canola seed cake. Int. J. Food Sci. Technol. 2015, 50, 1109-1115. [CrossRef]

32. Barba, F.J.; Parniakov, O.; Pereira, S.A.; Wiktor, A.; Grimi, N.; Boussetta, N.; Saraiva, J.A.; Raso, J.; Martin-Belloso, O.; WitrowaRajchert, D.; et al. Current applications and new opportunities for the use of pulsed electric fields in food science and industry. Food Res. Int. 2015, 77, 773-798. [CrossRef]

33. Moreira, S.A.; Alexandre, E.M.C.; Pintado, M.; Saraiva, J.A. Effect of emergent non-thermal extraction technologies on bioactive individual compounds profile from different plant materials. Food Res. Int. 2019, 115, 177-190. [CrossRef]

34. Alexandre, E.M.C.; Moreira, S.A.; Pintado, M.; Saraiva, J.A. Emergent extraction technologies to valorize fruit and vegetable residues. In Agricultural Research Updates; Gorawala, P., Mandhatri, S., Eds.; Nova Science: New York, NY, USA, 2017; Volume 17, pp. 37-79. ISBN 9781536109078.

35. Ricci, A.; Parpinello, G.P.; Versari, A. Recent Advances and Applications of Pulsed Electric Fields (PEF) to Improve Polyphenol Extraction and Color Release during Red Winemaking. Beverages 2018, 4, 18. [CrossRef]

36. Papachristou, I.; Akaberi, S.; Silve, A.; Navarro-López, E.; Wüstner, R.; Leber, K.; Nazarova, N.; Müller, G.; Frey, W. Analysis of the lipid extraction performance in a cascade process for Scenedesmus almeriensis biorefinery. Biotechnol. Biofuels 2021, 14, 1-14. [CrossRef]

37. Nowacka, M.; Tappi, S.; Wiktor, A.; Rybak, K.; Miszczykowska, A.; Czyzewski, J.; Drozdzal, K.; Witrowa-Rajchert, D.; Tylewicz, $\mathrm{U}$. The impact of pulsed electric field on the extraction of bioactive compounds from beetroot. Foods 2019, 8, 244. [CrossRef] [PubMed]

38. Moradi, N.; Rahimi, M. Effect of simultaneous ultrasound/pulsed electric field pretreatments on the oil extraction from sunflower seeds. Sep. Sci. Technol. 2018, 53, 2088-2099. [CrossRef]

39. Suryanto, H.; Fikri, A.A.; Permanasari, A.A.; Yanuhar, U.; Sukardi, S. Pulsed Electric Field Assisted Extraction of Cellulose From Mendong Fiber (Fimbristylis globulosa) and its Characterization. J. Nat. Fibers 2018, 15, 406-415. [CrossRef]

40. Yongguang, Y.; Yuzhu, H.; Yong, H. Pulsed electric field extraction of polysaccharide from Rana temporaria chensinensis David. Int. J. Pharm. 2006, 312, 33-36. [CrossRef]

41. Arshad, R.N.; Abdul-Malek, Z.; Roobab, U.; Qureshi, M.I.; Khan, N.; Ahmad, M.H.; Liu, Z.W.; Aadil, R.M. Effective valorization of food wastes and by-products through pulsed electric field: A systematic review. J. Food Process. Eng. 2021, 44. [CrossRef] 
42. Zia, S.; Khan, M.R.; Shabbir, M.A.; Aslam Maan, A.; Khan, M.K.I.; Nadeem, M.; Khalil, A.A.; Din, A.; Aadil, R.M. An Inclusive Overview of Advanced Thermal and Nonthermal Extraction Techniques for Bioactive Compounds in Food and Food-related Matrices. Food Rev. Int. 2020. [CrossRef]

43. Li, M.; Lin, J.; Chen, J.; Fang, T. Pulsed Electric Field-Assisted Enzymatic Extraction of Protein from Abalone (Haliotis Discus Hannai Ino) Viscera. J. Food Process. Eng. 2016, 39, 702-710. [CrossRef]

44. Lin, S.; Wang, L.; Jones, G.; Trang, H.; Yin, Y.; Liu, J. Optimized extraction of calcium malate from eggshell treated by PEF and an absorption assessment in vitro. Int. J. Biol. Macromol. 2012, 50, 1327-1333. [CrossRef] [PubMed]

45. Zhao, Y.; Zheng, Y.; He, H.; Sun, Z.; Li, A. Effective aluminum extraction using pressure leaching of bauxite reaction residue from coagulant industry and leaching kinetics study. J. Environ. Chem. Eng. 2021, 9, 104770. [CrossRef]

46. Zderic, A.; Zondervan, E. Polyphenol extraction from fresh tea leaves by pulsed electric field: A study of mechanisms. Chem. Eng. Res. Des. 2016, 109, 586-592. [CrossRef]

47. Kokosa, J.M. Selecting an extraction solvent for a greener liquid phase microextraction (LPME) mode-based analytical method. TrAC Trends Anal. Chem. 2019, 118, 238-247. [CrossRef]

48. Efthymiopoulos, I.; Hellier, P.; Ladommatos, N.; Russo-Profili, A.; Eveleigh, A.; Aliev, A.; Kay, A.; Mills-Lamptey, B. Influence of solvent selection and extraction temperature on yield and composition of lipids extracted from spent coffee grounds. Ind. Crops Prod. 2018, 119, 49-56. [CrossRef]

49. Pintać, D.; Majkić, T.; Torović, L.; Orčić, D.; Beara, I.; Simin, N.; Mimica-Dukić, N.; Lesjak, M. Solvent selection for efficient extraction of bioactive compounds from grape pomace. Ind. Crop. Prod. 2018, 111, 379-390. [CrossRef]

50. Pintać, D.; Majkić, T.; Torović, L.; Orčić, D.; Beara, I.; Simin, N.; Mimica-Dukić, N.; Lesjak, M. Extraction of Bioactive Compound from Some Fruits and Vegetables (Pomegranate Peel, Carrot and Tomato). Am. J. Food Nutr. 2016, 4, 8-19. [CrossRef]

51. Altemimi, A.; Lakhssassi, N.; Baharlouei, A.; Watson, D.G.; Lightfoot, D.A. Phytochemicals: Extraction, isolation, and identification of bioactive compounds from plant extracts. Plants 2017, 6, 42. [CrossRef]

52. Tiwari, B.K. Ultrasound: A clean, green extraction technology. TrAC-Trends Anal. Chem. 2015, 71, 100-109. [CrossRef]

53. Rodríguez-Pérez, C.; Quirantes-Piné, R.; Fernández-Gutiérrez, A.; Segura-Carretero, A. Optimization of extraction method to obtain a phenolic compounds-rich extract from Moringa oleifera Lam leaves. Ind. Crops Prod. 2015, 66, 246-254. [CrossRef]

54. Heleno, S.A.; Diz, P.; Prieto, M.A.; Barros, L.; Rodrigues, A.; Barreiro, M.F.; Ferreira, I.C.F.R. Optimization of ultrasound-assisted extraction to obtain mycosterols from Agaricus bisporus L. by response surface methodology and comparison with conventional Soxhlet extraction. Food Chem. 2016, 197, 1054-1063. [CrossRef]

55. Leong, S.Y.; Burritt, D.J.; Oey, I. Evaluation of the anthocyanin release and health-promoting properties of Pinot Noir grape juices after pulsed electric fields. Food Chem. 2016, 196, 833-841. [CrossRef] [PubMed]

56. Shree, T.J.; Sree, V.G.; Sundararajan, R. Enhancement of Bioactive Compounds from Green Grapes Extract using Pulsed Electric Field treatment. J. Cancer Prev. Curr. Res. 2018, 9. [CrossRef]

57. Lamanauskas, N.; Pataro, G.; Bobinas, Č.; Šatkauskas, S.; Viškelis, P.; Bobinaitè, R.; Ferrari, G. Impulsinio elektrinio lauko ittaka sulčiu ir bioaktyvių medžiagu išgavimui iš aviečiu ir ju produktų. Zemdirbyste 2016, 103, 83-90. [CrossRef]

58. Barba, F.J.; Brianceau, S.; Turk, M.; Boussetta, N.; Vorobiev, E. Effect of Alternative Physical Treatments (Ultrasounds, Pulsed Electric Fields, and High-Voltage Electrical Discharges) on Selective Recovery of Bio-compounds from Fermented Grape Pomace. Food Bioprocess. Technol. 2015, 8, 1139-1148. [CrossRef]

59. Liu, Z.W.; Zeng, X.A.; Ngadi, M. Enhanced extraction of phenolic compounds from onion by pulsed electric field (PEF). J. Food Process. Preserv. 2018, 42. [CrossRef]

60. Fincan, M. Extractability of phenolics from spearmint treated with pulsed electric field. J. Food Eng. 2015, 162, 31-37. [CrossRef]

61. Sarkis, J.R.; Boussetta, N.; Blouet, C.; Tessaro, I.C.; Marczak, L.D.F.; Vorobiev, E. Effect of pulsed electric fields and high voltage electrical discharges on polyphenol and protein extraction from sesame cake. Innov. Food Sci. Emerg. Technol. 2015, 29, 170-177. [CrossRef]

62. Xue, D.; Farid, M.M. Pulsed electric field extraction of valuable compounds from white button mushroom (Agaricus bisporus). Innov. Food Sci. Emerg. Technol. 2015, 29, 178-186. [CrossRef]

63. Frontuto, D.; Carullo, D.; Harrison, S.M.; Brunton, N.P.; Ferrari, G.; Lyng, J.G.; Pataro, G. Optimization of Pulsed Electric Fields-Assisted Extraction of Polyphenols from Potato Peels Using Response Surface Methodology. Food Bioprocess. Technol. 2019, 12, 1708-1720. [CrossRef]

64. Puértolas, E.; Martínez De Marañón, I. Olive oil pilot-production assisted by pulsed electric field: Impact on extraction yield, chemical parameters and sensory properties. Food Chem. 2015, 167, 497-502. [CrossRef] [PubMed]

65. Pashazadeh, B.; Elhamirad, A.H.; Hajnajari, H.; Sharayei, P.; Armin, M. Optimization of the pulsed electric field -assisted extraction of functional compounds from cinnamon. Biocatal. Agric. Biotechnol. 2020, 23, 101461. [CrossRef]

66. Mahalleh, A.A.; Sharayei, P.; Mortazavi, S.A.; Azarpazhooh, E.; Niazmand, R. Optimization of the pulsed electric field-assisted extraction of functional compounds from nepeta binaludensis. Agric. Eng. Int. CIGR J. 2019, 21, 184-194.

67. Pataro, G.; Carullo, D.; Ferrari, G. Effect of PEF pre-treatment and extraction temperature on the recovery of carotenoids from tomato wastes. Chem. Eng. Trans. 2019, 75, 139-144. [CrossRef]

68. Pataro, G.; Carullo, D.; Bobinaite, R.; Donsì, G.; Ferrari, G. Improving the extraction yield of juice and bioactive compounds from sweet cherries and their by-products by pulsed electric fields. Chem. Eng. Trans. 2017, 57, 1717-1722. [CrossRef] 
69. Siddeeg, A.; Manzoor, M.F.; Ahmad, M.H.; Ahmad, N.; Ahmed, Z.; Khan, M.K.I.; Maan, A.A.; Mahr-Un-Nisa; Zeng, X.A.; Ammar, A.F. Pulsed electric field-assisted ethanolic extraction of date palm fruits: Bioactive compounds, antioxidant activity and physicochemical properties. Processes 2019, 7, 585. [CrossRef]

70. Moghaddam, T.N.; Elhamirad, A.H.; Saeidi Asl, M.R.; Shahidi Noghabi, M. Pulsed electric field-assisted extraction of phenolic antioxidants from tropical almond red leaves. Chem. Pap. 2020, 74, 3957-3961. [CrossRef]

71. Bobinaite, R.; Pataro, G.; Lamanauskas, N.; Šatkauskas, S.; Viškelis, P.; Ferrari, G. Application of pulsed electric field in the production of juice and extraction of bioactive compounds from blueberry fruits and their by-products. J. Food Sci. Technol. 2015, 52, 5898-5905. [CrossRef]

72. Bobinaitè, R.; Pataro, G.; Visockis, M.; Bobinas, Č.; Ferrari, G.; Viškelis, P. Potential application of pulsed electric fields to improve the recovery of bioactive compounds from sour cherries and their by-products. In Proceedings of the 11th Baltic Conference on Food Science and Technology, Jelgava, Latvia, 27-28 April 2017; pp. 70-74. [CrossRef]

73. Niu, D.; Ren, E.F.; Li, J.; Zeng, X.A.; Li, S.L. Effects of pulsed electric field-assisted treatment on the extraction, antioxidant activity and structure of naringin. Sep. Purif. Technol. 2021, 265, 118480. [CrossRef]

74. Lamanauskas, N.; Bobinaitè, R.; Šatkauskas, S.; Viškelis, P.; Pataro, G.; Ferrari, G. Sulčiu spaudimas iš sušaldytu/atšildytu mèlyniu taikant impulsinio elektrinio lauko metoda. Zemdirbyste 2015, 102, 59-66. [CrossRef]

75. Saini, A.; Panesar, P.S.; Bera, M.B. Valorization of fruits and vegetables waste through green extraction of bioactive compounds and their nanoemulsions-based delivery system. Bioresour. Bioprocess. 2019, 6. [CrossRef]

76. Rudra, S.G.; Nishad, J.; Jakhar, N.; Kaur, C. Food Industry Waste: Mine of Nutraceuticals. Int. J. Sci. Enviro. Technol. 2015, 4, 205-229.

77. Sagar, N.A.; Pareek, S.; Sharma, S.; Yahia, E.M.; Lobo, M.G. Fruit and Vegetable Waste: Bioactive Compounds, Their Extraction, and Possible Utilization. Compr. Rev. Food Sci. Food Saf. 2018, 17, 512-531. [CrossRef]

78. Medina-Meza, I.G.; Barbosa-Cánovas, G.V. Assisted extraction of bioactive compounds from plum and grape peels by ultrasonics and pulsed electric fields. J. Food Eng. 2015, 166, 268-275. [CrossRef]

79. El Kantar, S.; Boussetta, N.; Lebovka, N.; Foucart, F.; Rajha, H.N.; Maroun, R.G.; Louka, N.; Vorobiev, E. Pulsed electric field treatment of citrus fruits: Improvement of juice and polyphenols extraction. Innov. Food Sci. Emerg. Technol. 2018, 46, 153-161. [CrossRef]

80. Irfan, S.; Ranjha, M.M.A.N.; Mahmood, S.; Saeed, W.; Alam, M.Q. Lemon Peel: A Natural Medicine. Int. J. Biotechnol. Allied Fields 2018, 7, 185-194.

81. Irfan, S.; Ranjha, M.M.A.N.; Mahmood, S.; Mueen-ud-Din, G.; Rehman, S.; Saeed, W.; Qamrosh Alam, M.; Mahvish Zahra, S.; Yousaf Quddoos, M.; Ramzan, I.; et al. A Critical Review on Pharmaceutical and Medicinal Importance of Ginger. Acta Sci. Nutr. Health 2019, 3, 78-82.

82. Faniyi, T.O.; Adewumi, M.K.; Jack, A.A.; Adegbeye, M.J.; Elghandour, M.M.M.Y.; Barbabosa- Pliego, A.; Salem, A.Z.M. Extracts of herbs and spices as feed additives mitigate ruminal methane production and improve fermentation characteristics in West African Dwarf sheep. Trop. Anim. Health Prod. 2021, 53, 312. [CrossRef]

83. Valdés, A.; Mellinas, A.C.; Ramos, M.; Burgos, N.; Jiménez, A.; Garrigós, M.C. Use of herbs, spices and their bioactive compounds in active food packaging. RSC Adv. 2015, 5, 40324-40335. [CrossRef]

84. Roselló-Soto, E.; Parniakov, O.; Deng, Q.; Patras, A.; Koubaa, M.; Grimi, N.; Boussetta, N.; Tiwari, B.K.; Vorobiev, E.; Lebovka, N.; et al. Application of Non-conventional Extraction Methods: Toward a Sustainable and Green Production of Valuable Compounds from Mushrooms. Food Eng. Rev. 2016, 8, 214-234. [CrossRef]

85. Giacometti, J.; Bursać Kovačević, D.; Putnik, P.; Gabrić, D.; Bilušić, T.; Krešić, G.; Stulić, V.; Barba, F.J.; Chemat, F.; Barbosa-Cánovas, G.; et al. Extraction of bioactive compounds and essential oils from mediterranean herbs by conventional and green innovative techniques: A review. Food Res. Int. 2018, 113, 245-262. [CrossRef]

86. Zhang, Q.W.; Lin, L.G.; Ye, W.C. Techniques for extraction and isolation of natural products: A comprehensive review. Chin. Med. 2018, 13. [CrossRef]

87. Segovia, F.J.; Luengo, E.; Corral-Pérez, J.J.; Raso, J.; Almajano, M.P. Improvements in the aqueous extraction of polyphenols from borage (Borago officinalis L.) leaves by pulsed electric fields: Pulsed electric fields (PEF) applications. Ind. Crop. Prod. 2015, 65, 390-396. [CrossRef]

88. Barba, F.J.; Grimi, N.; Vorobiev, E. Evaluating the potential of cell disruption technologies for green selective extraction of antioxidant compounds from Stevia rebaudiana Bertoni leaves. J. Food Eng. 2015, 149, 222-228. [CrossRef]

89. Gagneten, M.; Leiva, G.; Salvatori, D.; Schebor, C.; Olaiz, N. Optimization of Pulsed Electric Field Treatment for the Extraction of Bioactive Compounds from Blackcurrant. Food Bioprocess. Technol. 2019, 12, 1102-1109. [CrossRef]

90. Liu, Z.; Esveld, E.; Vincken, J.P.; Bruins, M.E. Pulsed Electric Field as an Alternative Pre-treatment for Drying to Enhance Polyphenol Extraction from Fresh Tea Leaves. Food Bioprocess. Technol. 2019, 12, 183-192. [CrossRef]

91. Hendrawan, Y.; Sabrinauly, S.; Hawa, L.C.; Rachmawati, M.; Argo, B.D. Analysis of the phenol and flavonoid content from basil leaves (Ocimum americanum L) extract using pulsed electric field (PEF) pre-treatment. Agric. Eng. Int. CIGR J. 2019, 21, 149-158.

92. Mushtaq, A.; Roobab, U.; Denoya, G.I.; Inam-Ur-Raheem, M.; Gullón, B.; Lorenzo, J.M.; Barba, F.J.; Zeng, X.A.; Wali, A.; Aadil, R.M. Advances in green processing of seed oils using ultrasound-assisted extraction: A review. J. Food Process. Preserv. 2020, 44, e14740. [CrossRef]

93. Yusuf, A.K. A Review of Methods Used for Seed Oil Extraction. Int. J. Sci. Res. 2017, 8, 1854-1861. 
94. Haji-Moradkhani, A.; Rezaei, R.; Moghimi, M. Optimization of pulsed electric field-assisted oil extraction from cannabis seeds. J. Food Process. Eng. 2019, 42, e13028. [CrossRef]

95. Batool, M.; Kauser, S.; Nadeem, H.R.; Perveen, R.; Irfan, S.; Siddiqa, A.; Shafique, B.; Zahra, S.M.; Waseem, M.; Khalid, W.; et al. A Critical Review on Alpha Tocopherol: Sources, RDA and Health Benefits. J. Appl. Pharm. 2020, 12, 19-39.

96. Chemat, F.; Rombaut, N.; Sicaire, A.G.; Meullemiestre, A.; Fabiano-Tixier, A.S.; Abert-Vian, M. Ultrasound assisted extraction of food and natural products. Mechanisms, techniques, combinations, protocols and applications. A review. Ultrason. Sonochem. 2017, 34, 540-560. [CrossRef] [PubMed]

97. Vorobiev, E.; Lebovka, N. Pulsed electric energy-assisted biorefinery of oil crops and residues. In Handbook of Electroporation; Miklavčič, D., Ed.; Springer: Cham, Switzerland, 2017; Volume 4, pp. 2863-2881. ISBN 9783319328867.

98. Sitzmann, W.; Vorobiev, E.; Lebovka, N. Applications of electricity and specifically pulsed electric fields in food processing: Historical backgrounds. Innov. Food Sci. Emerg. Technol. 2016, 37, 302-311. [CrossRef]

99. Shorstkii, I.; Koshevoi, E. Extraction Kinetic of Sunflower Seeds Assisted by Pulsed Electric Fields. Iran. J. Sci. Technol. Trans. A Sci. 2019, 43, 813-817. [CrossRef]

100. Zhou, Y.J.; Xue, C.M.; Zhang, S.S.; Yao, G.M.; Zhang, L.; Wang, S.J. Effects of high intensity pulsed electric fields on yield and chemical composition of rose essential oil. Int. J. Agric. Biol. Eng. 2017, 10, 295-301. [CrossRef]

101. Shorstkii, I.; Mirshekarloo, M.S.; Koshevoi, E. Application of Pulsed Electric Field for Oil Extraction from Sunflower Seeds: Electrical Parameter Effects on Oil Yield. J. Food Process. Eng. 2017, 40. [CrossRef]

102. Tamborrino, A.; Urbani, S.; Servili, M.; Romaniello, R.; Perone, C.; Leone, A. Pulsed electric fields for the treatment of olive pastes in the oil extraction process. Appl. Sci. 2020, 10, 114. [CrossRef]

103. Martínez, J.M.; Delso, C.; Álvarez, I.; Raso, J. Pulsed electric field-assisted extraction of valuable compounds from microorganisms. Compr. Rev. Food Sci. Food Saf. 2020, 19, 530-552. [CrossRef] [PubMed]

104. Yellamanda, B.; Vijayalakshmi, M.; Kavitha, A.; Reddy, D.K.; Venkateswarlu, Y. Extraction and bioactive profile of the compounds produced by Rhodococcus sp. VLD-10. 3 Biotech. 2016, 6, 261. [CrossRef]

105. Martínez, J.M.; Luengo, E.; Saldaña, G.; Álvarez, I.; Raso, J. C-phycocyanin extraction assisted by pulsed electric field from Artrosphira platensis. Food Res. Int. 2017, 99, 1042-1047. [CrossRef] [PubMed]

106. Jaeschke, D.P.; Mercali, G.D.; Marczak, L.D.F.; Müller, G.; Frey, W.; Gusbeth, C. Extraction of valuable compounds from Arthrospira platensis using pulsed electric field treatment. Bioresour. Technol. 2019, 283, 207-212. [CrossRef]

107. Martínez, J.M.; Delso, C.; Álvarez, I.; Raso, J. Pulsed electric field permeabilization and extraction of phycoerythrin from Porphyridium cruentum. Algal Res. 2019, 37, 51-56. [CrossRef]

108. Carullo, D.; Abera, B.D.; Casazza, A.A.; Donsì, F.; Perego, P.; Ferrari, G.; Pataro, G. Effect of pulsed electric fields and high pressure homogenization on the aqueous extraction of intracellular compounds from the microalgae Chlorella vulgaris. Algal Res. 2018, 31, 60-69. [CrossRef]

109. Silve, A.; Kian, C.B.; Papachristou, I.; Kubisch, C.; Nazarova, N.; Wüstner, R.; Leber, K.; Strässner, R.; Frey, W. Incubation time after pulsed electric field treatment of microalgae enhances the efficiency of extraction processes and enables the reduction of specific treatment energy. Bioresour. Technol. 2018, 269, 179-187. [CrossRef]

110. Nandhu Lal, A.M.; Prince, M.V.; Sreeja, R. Studies on Characterisation of Combined Pulsed Electric Field and Microwave Extracted Pectin from Jack Fruit Rind and Core. Int. J. Curr. Microbiol. Appl. Sci. 2020, 9, 2371-2380. [CrossRef]

111. Pataro, G.; Carullo, D.; Ferrari, G. PEF-assisted supercritical CO2 extraction of pigments from microalgae nannochloropsis oceanica in a continuous flow system. Chem. Eng. Trans. 2019, 74, 97-102. [CrossRef] 